\title{
3D STED Microscopy with Pulsed and Continuous Wave Lasers
}

\author{
Dissertation \\ zur Erlangung des Doktorgrades \\ der Mathematisch-Naturwissenschaftlichen Fakultäten \\ der Georg-August-Universität zu Göttingen
}

\author{
vorgelegt von \\ Benjamin Harke \\ aus Celle
}

Göttingen, den 20.02.2008 
D7

Referent: Prof. Dr. M. Münzenberg

Koreferent: Prof. Dr. S. W. Hell

Tag der mündlichen Prüfung: 


\section{D STED Microscopy with Pulsed and Continuous Wave Lasers}

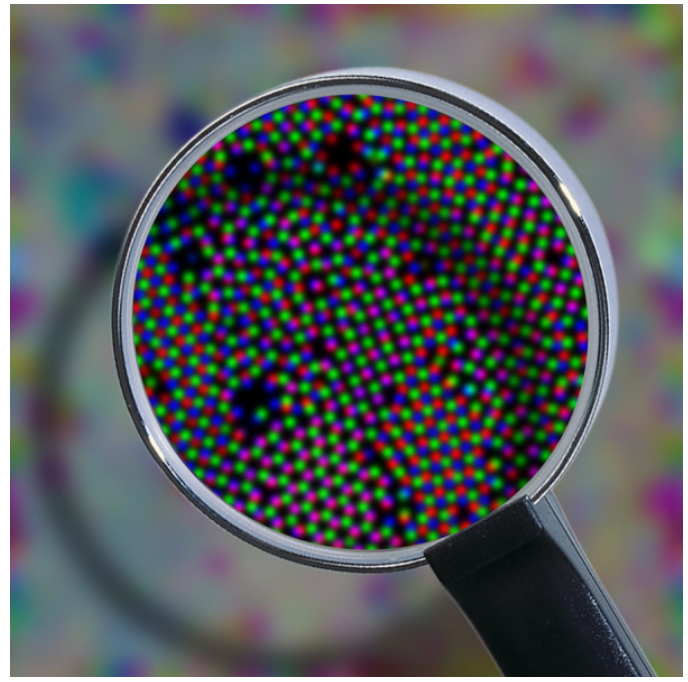




\section{Contents}

1 Introduction 1

1.1 Motivation . . . . . . . . . . . . . . . . . . . 1

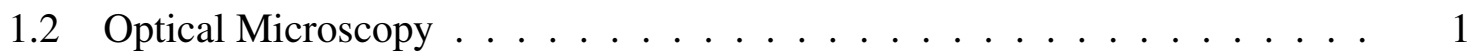

1.2 .1 Breaking the Diffraction Barrier $\ldots \ldots \ldots \ldots \ldots \ldots$

1.2 .2 STED Microscopy $\ldots \ldots \ldots \ldots \ldots \ldots \ldots \ldots$

\section{Quantitative Investigation of Resolution Enhancement with STED Mi-} croscopy

2.1 High Resolution with High Quality - in Search of the Best Depletion Pattern 8

2.2 Theoretical description of the resolution in a STED microscope - the square

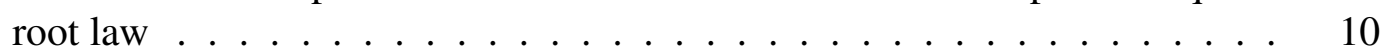

2.3 Experimental Validation of the Resolution Concept $\ldots \ldots \ldots \ldots$. . . . . 13

2.3 .1 Experimental Setup . . . . . . . . . . . . . . . . . . . . . 13

2.3 .2 Resolution Measurements . . . . . . . . . . . . . . . . . . 14

2.3 .3 The Quality of the Minimum . . . . . . . . . . . . . . . . . . . 19

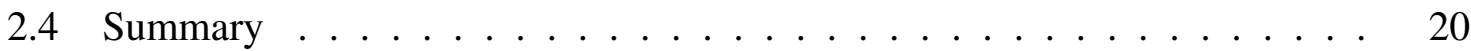

3 3D Imaging - Resolution Enhancement in All Spatial Directions 22

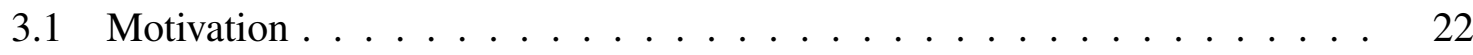

3.2 Experimental Results . . . . . . . . . . . . . . . . . . . 23

3.2 .1 Experimental Setup . . . . . . . . . . . . . . . . . . 23

3.2 .2 The Focal Volume . . . . . . . . . . . . . . . . . . . . . 24

3.2 .3 Three-Dimensional Nanoscopy of Colloidal Crystals . . . . . . . . 27

3.2 .4 Three-Dimensional Measurements in Biological Samples. . . . . . 32

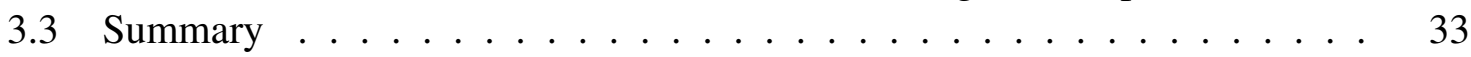

4 STED Microscopy with Continuous Wave Lasers 35

4.1 Simplify the Setup with CW - Motivation $\ldots \ldots \ldots \ldots . \ldots . \ldots 35$

$4.2 \quad$ A Theoretical Approach to CW STED . . . . . . . . . . . . . . . . . . 36

4.3 Experimental Results . . . . . . . . . . . . . . . . . . . . . . . 37

$4.3 .1 \quad$ Experimental Setup . . . . . . . . . . . . . . . . . . . . . . . . . . . . 37

4.3 .2 First Depletion Measurements . . . . . . . . . . . . . . . . . 38

$4.3 .3 \quad$ High Resolution Measurements with CW STED . . . . . . . . . . 39

$4.3 .4 \quad$ High Resolution Measurements in Biological Samples . . . . . . . 40

4.4 Discussion and Summary $\ldots \ldots \ldots \ldots \ldots$ 
5 Conclusion and Outlook 45

\begin{tabular}{ll}
\hline Bibliography & 48
\end{tabular}

\begin{tabular}{ll}
\hline A Appendix & 54
\end{tabular}

A.1 Measurement Parameters . . . . . . . . . . . . . . . . . 54

A.2 Measuring the Size of the Effective PSF . . . . . . . . . . . . . 54

A.3 STED Depletion Measurements $\ldots \ldots \ldots$. . . . . . . . . . . . . . . . . . . . . . . . . 55

A.4 Additional Measurements . . . . . . . . . . . . . . . . . . . 56

A.4.1 Microtubuli . . . . . . . . . . . . . . . 56

A.4.2 CW STED Microscopy . . . . . . . . . . . . . . . . . . 56

A.5 Sample Preparation . . . . . . . . . . . . . . . 56

\begin{tabular}{ll}
\hline B List of Publications & 60
\end{tabular} 


\section{Abbreviations}

$\begin{array}{ll}\text { 1D } & \text { one-dimensional } \\ \text { 2D } & \text { two-dimensional } \\ \text { three-dimensional } \\ \text { 4Pi-microscopy } & \text { microscopy using two opposing lenses in a coherent way } \\ \text { CW } & \text { continuous wave } \\ \text { Exc } & \text { excitation } \\ \text { Eff } & \text { effective } \\ \text { Det } & \text { detection } \\ \text { FWHM } & \text { full width at half maximum } \\ \Gamma & \text { time between two consecutive pulses } \\ h_{c} & \text { confocal PSF } \\ h_{\text {exc }} & \text { excitation PSF } \\ h_{\text {det }} & \text { detection PSF } \\ I_{S T E D} & \text { maximum intensity in the depletion focus } \\ I_{S T E D}(x) & \text { intensity near the depletion focus at position } x \\ k_{f l} & \text { decay rate for spontaneous emission }\left(k_{f l}=1 / \tau_{f l}\right) \\ \lambda_{S T E D} & \text { wavelength of the STED beam } \\ \lambda_{\text {exc }} & \text { wavelength of the excitation beam } \\ \eta & \text { fluorescence suppression by STED } \\ N A & \text { numerical aperture of a lens }(N A=n \text { sin } \alpha) \\ P_{2 D} & \text { helical phase mask (confines the confocal PSF in the lateral directions) } \\ P_{3 D} & \text { axial phase mask (confines the confocal PSF in all directions) } \\ \text { PSF } & \text { point spread function } \\ \text { RESOLFT } & \text { reversible saturable optical (fluorescence) transitions } \\ \text { SLM } & \text { spatial light modulator } \\ \text { STED } & \text { stimulated emission depletion } \\ \sigma_{S T E D} & \text { cross section for stimulated emission } \\ \tau & \text { pulse width in the pulsed mode } \\ \tau_{f l} & \text { fluorescence lifetime } \\ \text { Ti:Sa } & \text { Titanium:Sapphire } \\ \text { SNOM } & \text { scanning near-field optical microscopy } \\ \text { OPO } & \text { uptical parametric oscillator } \\ \text { UV } & \end{array}$




\section{Introduction}

\subsection{Motivation}

The microscope is probably one of the most powerful tools for scientists working in interdisciplinary fields. It gives the possibility to investigate a certain sample and retrieve information about the structure, the material, the shape or the size of the observed object. A very important parameter of the imaging technique is its resolution. It is commonly defined as the minimum distance between two small objects in the sample that can still be separated during the imaging process.

Most of the microscopy techniques can be classified into two groups: the near and the far field optical microscopy.

The near field microscopy has a very high resolution. The atomic force microscope for example scans a tiny tip over the sample surface and gets information about the structure of the surface with a resolution that mainly depends on the form of the scanning tip. Atomic resolution can be achieved with this imaging technique [1]. In scanning tunneling microscopy (which works with the same principle but is limited to conducting surfaces) [2] a conducting tip measures the tunneling current as the tip is moved over the surface. The tunnel current depends on the distance between the tip and the surface and provides information about the structure of the conducting sample. In a scanning optical microscope (SNOM, [3]) a small light source generated by a sub-wavelength aperture close to the sample surface is moved over the surface of the sample.

However, all methods of near field microscopy are limited to the sample surface. Even with a very high resolution in the axial direction (perpendicular to the surface), no information hidden below the surface can be extracted. Additionally, biological problems can not be solved because currently no marker molecules permit the required specificity.

In conventional far field microscopy, however, a resolution on the scale of the wavelength in all spatial directions can be achieved with a high penetration depth into the sample. It allows the user to use specific markers e.g. to label proteins for biological imaging. This work will focus on the far field optical microscopy whose basics will be discussed in the following section.

\subsection{Optical Microscopy}

Investigating samples by using focused light beams with high resolution, high specificity and high speed has been always a major focus for scientists from different research fields. Scientists worked intensively in these fields to read out information about the structure and 
dynamics of the sample. Ernst Abbe was one of the main pioneers working on the theory of light focusing. In the 19th century he worked together with the opto-mechanist Carl Zeiss and they developed the first microscope that was built according to the foregone calculations of Ernst Abbe. These calculations included the diffraction theory of light [4]. One of the most important result of Abbe's calculations was the fact that the size of a focal spot generated by a lens is diffraction limited to:

$$
\begin{aligned}
\Delta x, \Delta y & \approx \frac{\lambda}{2 \cdot n \cdot \sin (\alpha)} \\
\Delta z & \approx \frac{\lambda}{2 \cdot n \cdot \sin ^{2}\left(\frac{\alpha}{2}\right)}
\end{aligned}
$$

Here $x$ and $y$ correspond to the particular lateral directions and $z$ to the axial one, $\lambda$ is the wavelength of the irradiated light, $n$ is the refractive index of the medium and $\alpha$ the maximum angle of the irradiated light. With the definition of the numerical aperture, $N A=n \cdot \sin (\alpha)$, the size of the focus is mainly governed by the wavelength used and the numerical aperture of the optical system.

In optical microscopy the measured signal is generated in the focal spot region. Therefore the size of the focal spot gives the resolution of the microscope. As a result the limited size of the focus due to Abbe's formulas gives directly a limit for the resolution capability of the microscope.

Despite its limited resolution, optical microscopy is still one of the most important tools for biological scientists, because it has been technically optimized in terms of specificity and speed [5]. Labeling specific proteins is one of the most important benefits in fluorescence microscopy. One of the standard labeling techniques is the immunolabeling. A primary antibody is linked to the investigated protein. Secondary antibodies stained with dye molecules can now bind to the primary antibody. The advantage of such a labeling process in two steps is the enhanced fluorescence signal, because a bunch of dye molecules give the position of the protein. Alternatives to the labeling technique is the detection of fluorescent proteins [6][7] directly expressed by the cell. The simultaneously recording of multiple signals allows the localization of different molecules analyzing the interaction or co-localization of these contributing molecules.

Fast scanning techniques in laser scanning microscopes (LSM) offered the possibility of life cell imaging. Tracking single proteins in cells uncovers transport or signal transfer mechanisms.

However, to improve the resolution in far field microscopes relying only on linear light interactions, the extent of the focus determined by Abbe's law (1.1) must be reduced. The improvement can be realized by either decreasing the wavelength of the irradiated light or by increasing the numerical aperture of the objective lens.

For liquid immersion lenses the numerical aperture is technically limited to approximately 1.45. With solid immersion lenses [8] a numerical aperture of 2.0 [9] was achieved depending on the choice of the immersion medium. Due to complex experimental setup and 
high sensitivity toward slight refractive index changes, the solid immersion lenses are not convenient for biological imaging. For all standard commercial microscopes working with oil immersion lenses the numerical aperture is limited to 1.4.

To increase the resolution further, the aperture size of an optical system is effectively enlarged by using two identical lenses opposing each other. This has first been demonstrated in the 4Pi-microscopy [10][11] using interference effects to overcome the limited size of the focus in the axial direction. The coherently overlaid foci generate an interference pattern whose axial dimension is effectively reduced relative to the original focus size. Deconvolution algorithms can remove artifacts caused by the side lobes of the interference pattern. Optical sectioning with enhanced axial resolution of about $100 \mathrm{~nm}$ has been shown in many biological applications [12][13][14].

The commonly used wavelengths in far field microscopy are in the visible range between $450 \mathrm{~nm}$ and $700 \mathrm{~nm}$. This is based on the fact that visible light is not toxic for biological tissue and that it can be conveniently aligned since it is visible to the eye. Another reason is that the fluorescence wavelength of most of the fluorescent dyes is in this range. A reduction of the wavelength implicates problems for biological imaging. The absorption of near ultraviolet light (200 $\mathrm{nm}$ to $400 \mathrm{~nm}$ ) in the cell causes photodamage [15]. Also complex optics are needed for focusing UV-light which would cause difficulties in the realization of a UV microscope.

A tremendous reduction of the wavelength has been realized by using electron beams, whose De-Broglie wavelength is more than one order of magnitude lower than the wavelength of visible light. With this method, the resolution could be enhanced to the molecular level [16]. Selective staining in biological tissue can be achieved by immunolabeling proteins with gold particles as they give a contrast signal for the localization inside the cell. High photo destruction due to the high energy of the irradiated electrons makes this imaging techniques not viable for live cell imaging. The low penetration depth of the electron beam limits this method to the surface of the sample, which has been circumvented by imaging thin slices of $<100 \mathrm{~nm}$ of the sample [17] via transmission electron microscopy. But the electron microscope requires complex sample preparation as well as a complex experimental platform as the method only works in evacuated chambers. In any case, electron microscopy is still a standard imaging tool in biological and particularly in material sciences.

The confocal microscope [18] has overcome many of these mentioned difficulties. Working with visible light in room temperature and atmospheric pressure makes the sample preparation and the experimental setup straightforward. The confocal microscope is a type of fluorescence microscopy and offers the user to achieve optical sectioning [19] from the inside of the investigated sample with a suppressed background signal. A schematic layout of a confocal microscope is shown in figure 1.1. The excitation light is focused into the sample by an objective lens and the fluorescence is collected by the same lens and focused onto a point-like detector. The image data acquisition is realized by either scanning the focus through the sample or vice versa. The size of the detector is classified in units of backprojected Airy discs, which is defined as the lateral distance between the first two minima in a lateral profile of the generated light focus. The confocal point spread function (PSF) is defined as the probability of exciting a molecule at a particular position $\vec{r}$ multiplied with the 


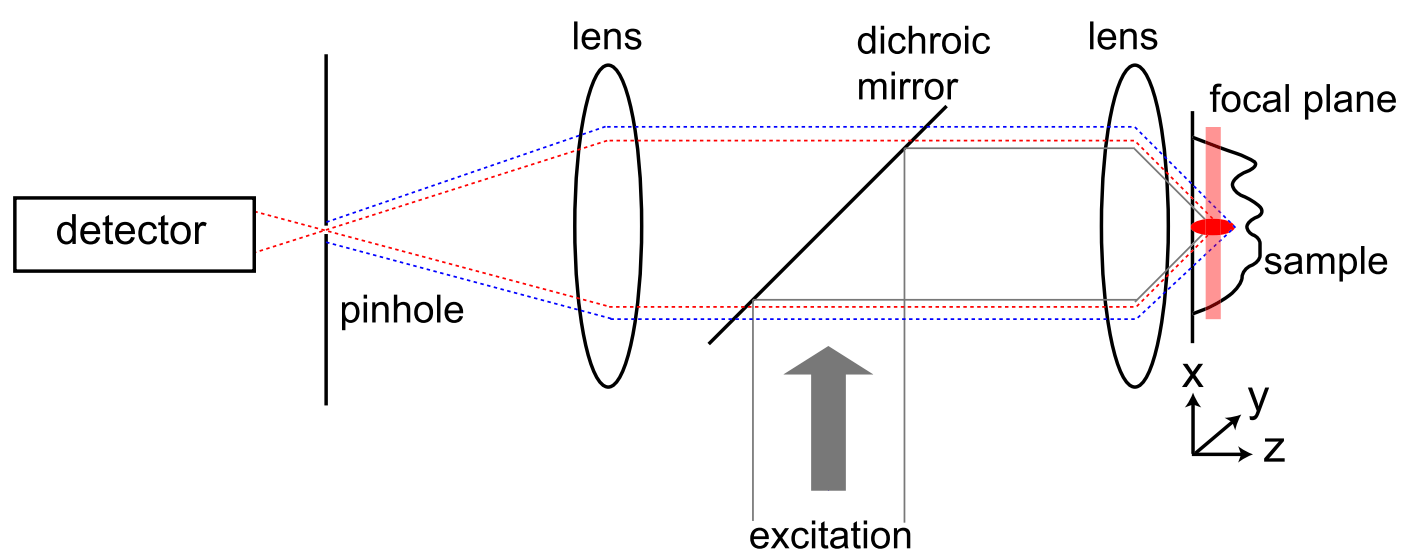

Figure 1.1: Schematic diagram of a confocal microscope

probability to detect the emitted photon in the detector:

$$
h_{c}(\vec{r})=h_{\text {exc }}(\vec{r}) h_{\text {det }}(\vec{r})
$$

With a detector size of below one Airy disc (means $h_{e x c} \approx h_{\text {det }}$ ) the product of both PSFs leads to a strong suppression of the out of focus signal. A pinhole blocks the signal not generated in the focal area and thus the latter is not detected making optical sectioning in scattering samples possible. Since it is implemented in most conventional fluorescence microscopes, the confocal microscopy is an imaging standard. Therefore it acts as a reference for all the improved data in this thesis.

Due to scattering effects causing a deformed and enlarged focus the penetration depth of a confocal microscope is limited [20].

\subsubsection{Breaking the Diffraction Barrier}

The microscopy techniques previously mentioned followed the Abbe's law (1.1) concerning the limited resolution capability. Several methods have come up to break or circumvent the diffraction barrier.

The two photon microscopy works with the non linear effect of two photon absorption. The molecules in the focal area are excited by absorbing two photons simultaneously with half of the required energy. The probability of such a transition depends on the square of the intensity of the irradiated light confining the focal spot even in high scattering samples to a defined volume and reduced background. But due to the doubled wavelength the resulting resolution of this microscope does not exceed the resolution of a confocal microscope [21]. With this two photon absorption a penetration depth of several $100 \mu \mathrm{m}$ in biological tissue is achievable [22] due to lower scattering effects of the higher wavelength. However, high intensities required for the excitation process cause higher photobleaching in the focal region whereas it is reduced in the surrounding where the dye can not be excited due to the two photon process. 
A recently introduced concept for breaking the diffraction barrier has already been successfully explored by using localization methods in a wide-field microscopy technique. It is based on single switchable fluorescent molecules. The activation intensity is set to a small value so that randomly only a few molecules in the imaging area (at most one molecule per PSF) are activated. By exciting these molecules the fluorescence signal can be read out on a CCD camera. The positions of the molecules can be localized with a high accuracy which mainly depends of the number of emitted photons per molecule, even though the size of the imaged fluorescent spot on the camera is diffraction limited. The activated molecules are switched off before the next set of molecules are activated. By iteratively reading out the signal of every molecule on the sample, the image can be completely reconstructed. With this method a resolution of below $40 \mathrm{~nm}$ has been measured [23][24][25]. This technique has been extended by implementing two photon activation of the molecules [26] or volume read out algorithms [27] to three dimensional samples. However, this method is more or less limited to photoswitchable molecules and achieving fast recording speeds required for live cell imaging is challenging.

A very successful concept of breaking the diffraction barrier has been realized in the RESOLFT-technique (reversible saturable optical fluorescence transition) [28]. In fluorescence microscopy, this concept circumvents the resolution limit by selectively switching between a bright state and a dark state in the outer region of the excitation focus. To achieve sub-diffraction resolution, two processes are required: the depletion of the bright state must be possible and the shape of the switching beam has to be modified to feature an intensity zero in its center.

Starting with such a two level system with the bright state A and a dark state B, the basic of this idea is to have a (mostly) light driven transition that quenches the molecule from the state A to state B. This transition has to be optically saturable. The deexcitation light should feature an intensity minimum in the center of the focal spot, which can be easily created by interference effects. The excited molecules in the outer region of the focus are then quenched to the dark state B. The result is an effective focal area containing molecules in the bright state A that is significantly smaller than the original one. With an increase of the deexcitation intensity, owing to the saturation effect, molecules closer to the center of the focus are increasingly pumped to the dark state. This results in a smaller effective focus and therefore in higher resolution. What makes this method so important in contrast to other previously shown methods is the fact, that no theoretical limit of the resolution is given. Starting with two diffraction-limited spots results in an effective focus whose size is mainly governed by the efficiency of the switching process. This depletion efficiency can directly be influenced by the intensity of the irradiated light or by the cross section for the transition.

Several publications have already validated this method. The reversible photoswitching of fluorescent proteins [29][30] showed the ability to image biological systems. Due to low required intensities, the method can be implemented in wide-field techniques allowing fast image acquisitions [31].

Using the long living triplet state as the dark state, the ground state depletion [32][33] showed that resolution enhancement in technical and biological samples can be performed with a simplified setup using just one light source with a given wavelength. Due to high rates 
for the transition from the excited singlet state to the triplet state, the dye molecules can be efficiently pumped into the long living dark state within a pump light distribution featuring a local minimum in the center of the excitation focus. The probe of the remaining fluorescence is performed by the same light source as for the pumping process resulting in a simple and inexpensive experimental setup.

The very first concept of RESOLFT was realized by STED microscopy which is explained in the following chapter.

\subsubsection{STED Microscopy}

STimulated Emission Depletion (STED) microscopy is part of the RESOLFT concept and was first proposed in 1994 by Hell and Wichmann [34]. In this concept the fluorescent state is depleted by stimulated emission [35].

In figure 1.2 a schematic energy level diagram shows the basic concept of the stimulated emission. The dye molecule is excited from the ground state $S_{0}$ to a higher vibrionic level of the first excited state $S_{1}^{v i b}$ by the excitation beam. In a very short time range (several $p s$ ) it decays non-radiatively to the lowest energy level of $S_{1}$. This excited molecule can relax to the ground state via spontaneous emission by sending out a photon which can be detected as the fluorescence signal because it is red shifted to the excitation wave length. However, the excited state of the molecule can also be quenched via stimulated emission. A second laser beam that matches the energy gap between $S_{1}$ and $S_{0}^{v i b}$ stimulates the molecule to relax to the ground state by emitting a photon with the same wavelength as the stimulated emission photon. This wavelength is not detected as a fluorescence signal, because it is shifted to a longer wavelength (depletion wavelength is set to the red part of the emission spectrum). The decay rate for spontaneous emission is defined as $k_{f l}$ and $k_{S T E D}$ for the stimulated emission. For an efficient quenching process the decay rate for stimulated emission has to be significantly larger than that for spontaneous emission, $k_{f l} \ll k_{S T E D}$. This can be realized by either increasing the intensity of the STED beam or by increasing the cross section for stimulated emission of the dye by optimizing the depletion wavelength. Because of the

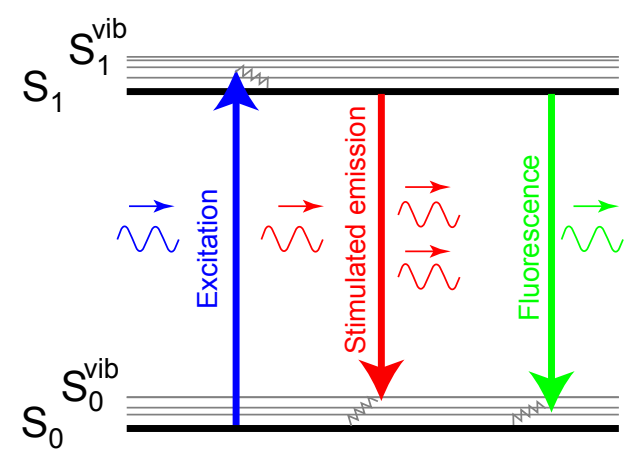

Figure 1.2: Schematic two-level energy diagram of a dye molecule. 
very fast decay of the higher vibrionic levels $\left(S_{0}^{v i b}\right)$ to the ground state $\left(S_{0}\right)$, the stimulated depletion laser can not excite the same molecule to the excited state $S_{1}^{v i b}$.

The first proof of the principle was performed by overlaying two spatially shifted foci to quench the fluorescence at one edge of the excitation focus [36]. Meanwhile the STED microscopy has become a popular tool for investigating biological and artificial structured samples. The effect of stimulated emission works in principle with every dye which makes this method so powerful. A resolution of $15 \mathrm{~nm}-20 \mathrm{~nm}$ in the lateral directions has been demonstrated in biological samples [37]. Combining this idea of stimulated emission depletion with the 4Pi-microscopy has lead to an axial resolution of below $40 \mathrm{~nm}$ [38][39]. In 2007 the first commercially available STED microscope was introduced onto the market (Leica Microsystem, Mannheim, Germany).

In any case, there are still open possibilities to improve the STED technique. The resolution capability in STED microscopy has already been shown in optical sections particularly in the lateral directions. Biological samples have been prepared in a manner, that the resolution enhancement in the lateral plane is sufficient to tackle biological problems. However, a cell for example is a three dimensional object and has to be recorded in all spatial directions in order to get information about structural details. The way to three dimensional imaging with an enhanced resolution in all directions will be presented in this work.

The influence of different depletion patterns on the resolution of the STED microscope is thereby a very important parameter. A quantitative investigation of the resolution enhancement in a STED microscope will be discussed in the following chapter.

The capability of performing three dimensional imaging will be presented in chapter 3. Thereby for the first time the incoherently combination of two depletion patterns - one for the resolution enhancement in the lateral plane and one for the axial direction - will be presented. The resulting minimized extent of the focal spot enables the three dimensional imaging of colloidal crystals with an enhanced resolution in all three directions.

Currently the implementation of STED microscopy as a standard imaging tool is mainly inhibited by the complex experimental platform including sophisticated and expensive laser sources as well as electrical equipment. In chapter 4, this thesis shows the first ever use of continuous wave (CW) lasers for the excitation and STED light sources. The simplicity of the setup can facilitate the way to a wide use of STED microscopy allowing in principle every existing scanning fluorescence microscope to perform high resolution. 


\section{Quantitative Investigation of Resolution Enhancement with STED Microscopy}

The fluorescence inhibition mechanism in the form of stimulated emission depletion has already been explained in chapter 1.2.2. Utilizing this mechanism for achieving resolution increase in microscopy will be explained in this chapter. Different optimal depletion patterns exist depending on the desired increase of resolution in one, two or three directions.

A general mathematical analysis of the resolution capability of a STED microscope will be derived in chapter 2.2 accompanied by experimental data that validates this mathematical description.

\subsection{High Resolution with High Quality - in Search of the Best Depletion Pattern}

The distribution of the depletion light has a strong influence on the quality of the measurement. An added phase retardation in the depletion beam forms the depletion pattern itself. The phase retardation can be realized by using a spatial light modulator (SLM) with the advantage of the high flexibility of this device; different patterns can be applied in a very short time. However, a disadvantage is the resulting complexity of the setup. The SLM has to be imaged into the back aperture of the lens, which results in extended beam paths. An alternative to the SLM are simple glass substrates, where the phase retardation is realized by adding denser optical material (Polymer, Cryolite) to the substrate. With the thickness of the material, the relative phase retardation can be aligned. For these very simple and inexpensive optical devices, no imaging into the aperture of the lens is required, which makes the setup more compact and stable. However, one loses the flexibility of using various phase patterns in a short time.

Figure 2.1 shows the most common phase masks used for depletion patterns with their corresponding distributions in the $x y$ and $x z$ planes. The colormaps of the images are normalized to the same maximum value. All different relative intensities for given depletion patterns are summarized in the accompanying table 2.1.

The most effective phase mask for the resolution enhancement in one direction is the mask $P_{1 D}$ that retards a semicircle of the back aperture of the lens by $\pi$. The corresponding pattern can reduce the size of the effective focus in one lateral direction and leaves the other 


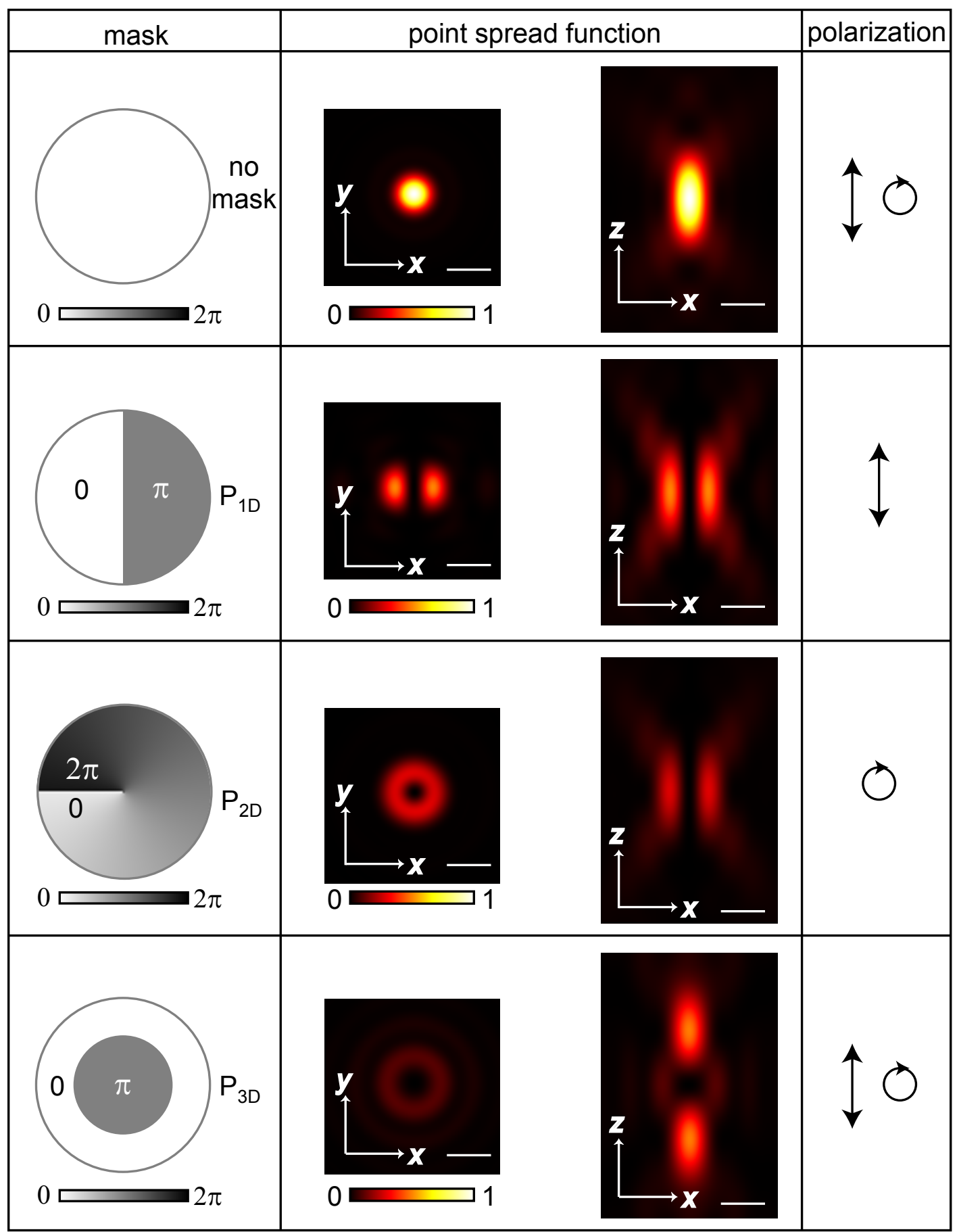

Figure 2.1: Different masks for the depletion beam yield different depletion PSFs. Scale bars $500 \mathrm{~nm}$. Calculated PSFs for the wavelength of $760 \mathrm{~nm}$ and a numerical aperture of 1.4. 


\begin{tabular}{|c|c|c|}
\hline mask & Max. Intensity in $x y$ plane & Max. Intensity in $x z$ plane \\
\hline \hline no mask & 1.00 & 1.00 \\
$P_{1 D}$ & 0.50 & 0.50 \\
$P_{2 D}$ & 0.33 & 0.33 \\
$P_{3 D}$ & 0.11 & 0.50 \\
\hline
\end{tabular}

Table 2.1: Relative maximal intensities of the intensity distributions in the $x y$ and $x z$ plane Numbers correlated to the PSFs shown in figure 2.1.

two directions unaltered [40][41]. For this phase mask, linearly polarized light is required to achieve an intensity zero at the center of the PSF. An incoherent combination of patterns produced by two phase masks of the latter type leads to an effective resolution enhancement in two directions, when these two phase plates are rotated by $90^{\circ}$ [40]. Though, due to polarization effects, the incoherent combination of the two pattern does not give a completely uniform resolution increase. An example of a measurement with a lateral resolution enhancement using this combination is shown in the appendix A.4.1.

The most effective mask for the resolution enhancement in both lateral directions is the mask $P_{2 D}$ that adds a helical phase retardation to the beam. It reduces the size of the effective focus uniformly in the two lateral directions, but does not change anything along the axial direction. Circularly polarized light with the same sense of rotation of the polarization vector as the phase mask itself $(0 \rightarrow 2 \pi)$ is required for this depletion mask to get a symmetric PSF with a high quality zero intensity at the center of the ring. In recent years, the only technique to generate such a helical phase retardation was the use of the SLM [42][43][44]. In this thesis a phase plate (vortex pattern, RPC Photonics, Rochester, NY, USA) for generating the depletion pattern was used for the first time. A polymer coated on the glass substrate generates the phase retardation. The maximal thickness of the polymer layer is adapted to the wavelength of the depletion light.

$P_{3 D}$ has a simple geometry that generates a very effective depletion pattern in all directions [41] [45][46]. The inner circle area of the back aperture of the lens is retarded by $\pi$ which results in a strong reduction in the axial direction, but also a moderate reduction in the lateral direction. This phase mask does not require a specific polarization.

The influence of the different phase masks on the resolution of a high resolution microscope will be discussed in the following section, while the possibility of incoherently combining two of the mentioned phase masks will be demonstrated in chapter 3 .

\subsection{Theoretical description of the resolution in a STED microscope - the square root law}

Stimulated emission depletion has been described in chapter 1.2.2 as well as the most effective phase masks for the best depletion pattern in the previous section. This section focuses on the resolution increase achievable by a STED microscope employing different 
phase masks.

Starting with a pulsed laser system, the excitation pulse and the STED pulse are separated in time; the excitation pulse is directly followed by the depletion pulse. The schematic energy diagram of the corresponding photo physical system has already been shown in figure 1.2. To get a quantitative description of the depletion process, the time dependence of the population $N_{1}$ of the first excited state $S_{1}$ has to be investigated. The differential equation for the state population of the two level system, with the rates $k_{f l}$ for the spontaneous emission and $k_{S T E D}$ for the simulated emission, is given by:

$$
\frac{d N_{1}(t)}{d t}=-\left(k_{f l}+k_{S T E D}\right) N_{1}(t)
$$

With the given initial conditions $N_{1}(0)=1$ and $N_{1}(t \rightarrow \propto)=0$, the solution of the equation is

$$
N_{1}(t)=e^{-\left(k_{f l}+k_{S T E D}\right) t} .
$$

Assuming a rectangular pulse with width $\tau$, the population $N_{1}$ can be interrogated just after the STED pulse by collecting the fluorescence. With $k_{S T E D}=\sigma_{S T E D} I_{S T E D}$, where $\sigma_{S T E D}$ describes the cross section for stimulated emission of the STED beam and $I_{S T E D}$ the depletion intensity of the STED beam, equation (2.2) results in

$$
N_{1}\left(I_{S T E D}\right)=e^{-\sigma_{S T E D} \tau I_{S T E D}+k_{f l}} .
$$

The suppression factor $\eta$ is defined as the reduced fluorescence signal relative to the absence of the depletion light:

$$
\eta\left(I_{S T E D}\right)=\frac{N_{1}\left(I_{S T E D}\right)}{N_{1}(0)}=\frac{e^{-\sigma_{S T E D} \tau I_{S T E D}+k_{f l}}}{e^{k_{f l}}}=e^{-\sigma_{S T E D} \tau I_{S T E D}} .
$$

Defining the saturation intensity $I_{S}$ where half of the excited molecules are quenched to the ground state, leads to an expression for $I_{S}$ :

$$
\eta\left(I_{S}\right)=1 / 2=e^{-\ln 2 \sigma_{S T E D} \tau I_{S}} \Longleftrightarrow I_{S}=\frac{\ln 2}{\tau \sigma_{S T E D}}
$$

To give a quantitative prediction of the resolution of a STED microscope, the shape of the depletion pattern has to be taken into account. Thereby at the central peak of the excitation focus, the depletion pattern can be written as a parabola in a particular spatial direction $x$ with a certain steepness $a, I_{S T E D}(x)=4 I_{S T E D} a^{2} x^{2} . I_{S T E D}(x)$ is defined as the intensity of the depletion PSF at the position $x$. In this case $I_{S T E D}$ is defined as the maximal intensity present in the focal distribution of the depletion beam.

To support this statement, figure 2.2 demonstrates two parabolic fits to the depletion pattern, generated by the phase mask $P_{2 D}$ (a) and $P_{3 D}$ (b). The good agreement of the red dashed fitted line to the intensity profile of the patterns confirms the idea of approximating $I_{S T E D}(x)$ as a parabola. 
(a)

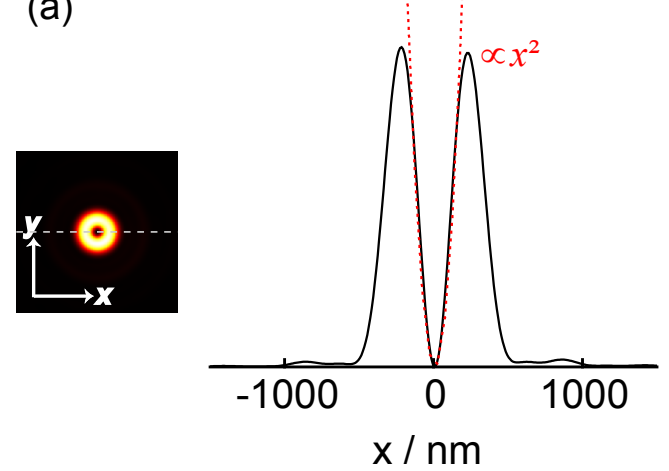

(b)
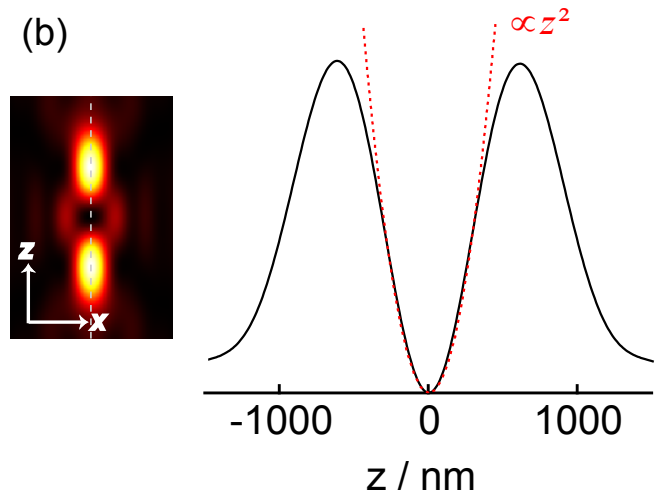

Figure 2.2: The parabola fit to the profiles along two different depletion patterns, generated with $P_{2 D}$ (a) and $P_{3 D}(\mathrm{~b})$.

To compare the resolution of the STED microscope $d$ to that of a confocal microscope $d_{c}$, the confocal PSF $h_{c}$ has to be taken into account. It can be described as a Gaussian function with width $d_{c}$ (in the lateral directions it is $\approx \lambda / 2 N A$ followed from the Abbe equations (1.1)). The FWHM of the central peak of the STED microscope is then given by this confocal PSF weighted with the suppression of the fluorescence, which is described by the equation (2.4):

$$
h_{e f f}(x)=h_{c}(x) \eta(x)=e^{-\frac{4 \cdot \ln 2 \cdot x^{2}}{d_{c}^{2}}} \cdot e^{-\frac{4 \cdot \ln 2 \cdot a^{2} I_{S T E D} \cdot x^{2}}{I_{S}}}=e^{-4 \cdot \ln 2 \cdot\left(d_{c}-2+a^{2} \frac{I_{S T E D}}{I_{S}}\right) \cdot x^{2}}
$$

This describes a Gaussian distribution with the FWHM $d$, that corresponds to the effective resolution of a STED microscope along a particular direction defined by $x$;

$$
d=\frac{d_{c}}{\sqrt{1+d_{c}^{2} a^{2} \varsigma}},
$$

$\varsigma$ is a parameter that describes the magnitude of the depletion effect of the STED beam, named saturation factor and defined as $\varsigma=\frac{I_{S T E D}}{I_{S}}$.

The resolution of a STED microscope can be increased to infinity, in principle, by increasing the saturation factor $\varsigma$. It is mainly governed by the saturation intensity, which is a dye characteristic constant, the applied STED intensity, the original confocal resolution and the shape of the depletion pattern, that enters the calculation through the steepness $a$ of the corresponding parabola.

To validate the approximations in equation (2.7), a calculation was performed by simulating the excitation and STED PSF [47]. With the given equations for the stimulated emission process, the FWHM of the effective PSF can be calculated. The result of this test is presented in figure 2.3. It shows the resolution of a STED microscope for the helical phase mask, $P_{2 D}$, on the left hand side as well as for the axial phase mask, $P_{3 D}$, on the right side. In both cases, the fit of equation (2.7) matches exactly the calculated points. The fit parameter $a$ and $d_{c}$ are written down in the figure. For the lateral phase mask, the steepness 

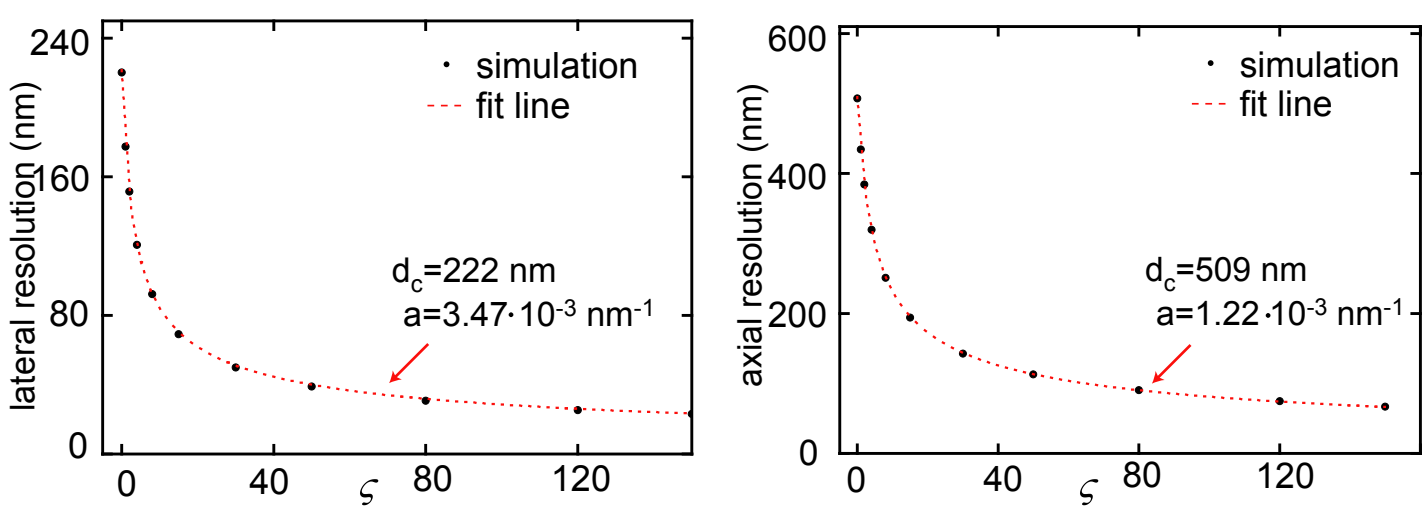

Figure 2.3: Theoretical resolution of a STED microscope for two different depletion patterns. The red dashed line is the fit line of the equation (2.7) to the data.

is $a_{P_{2 D}}=3.47 \cdot 10^{-3} \mathrm{~nm}^{-1}$ and for the axial phase plate $a_{P_{3 D}}=1.22 \cdot 10^{-3} \mathrm{~nm}^{-1}$. A comparison to the experimentally acquired data will be performed in the following section.

\subsection{Experimental Validation of the Resolution Concept}

\subsubsection{Experimental Setup}

The experimental setup used for the following measurements is shown in figure 2.4. The excitation beam is generated by a pulsed laser diode with a wavelength of $\lambda_{E x c}=635 \mathrm{~nm}$ and a pulse length of approximately $70 \mathrm{ps}$. The beam is aligned through a lens-pinhole (PH)lens system to enlarge the beam diameter. A dichroic mirror (DM1) reflects the beam and the latter is transmitted by a second dichroic mirror (DM2) before it is focused by an objective lens (OL, 100x NA 1.40 oil immersion, PL APO, Leica Microsystems). The sample is fixed on a piezo-scanning stage that can be scanned in all three spatial directions with a positioning accuracy of $5 \mathrm{~nm}$. The fluorescence emitted by the sample passes the two dichroic mirrors (DM1 and DM2) and is focused by the tube lens (TL) onto a multi mode fiber connected to a single photon avalanche photo detector (APD, Perkin Elmer, USA), which acts like a pinhole with a size corresponding to 0.7 times magnified Airy disc. For measuring samples with a very high brightness $(>1 \mathrm{MHz}$ ), the fiber is replaced with a fiber splitter dividing the signal into 4 fibers connected to different APDs. With these components, the setup of a standard confocal microscope is realized.

The STED beam is generated by a Titanium-Sapphire-laser (Ti:Sa, MIRA 900, Coherent, USA). The light of this laser is coupled into 100 meters of a polarization maintaining single mode fiber (PMF) to stretch the pulse from the initial width of approximately $100 \mathrm{fs}$ to about $300 \mathrm{ps}$. In order to avoid any nonlinear effects during the incoupling process, the light was prestretched by guiding through about $1 \mathrm{~m}$ of glass rods (SF6) in front of the fiber coupler. 


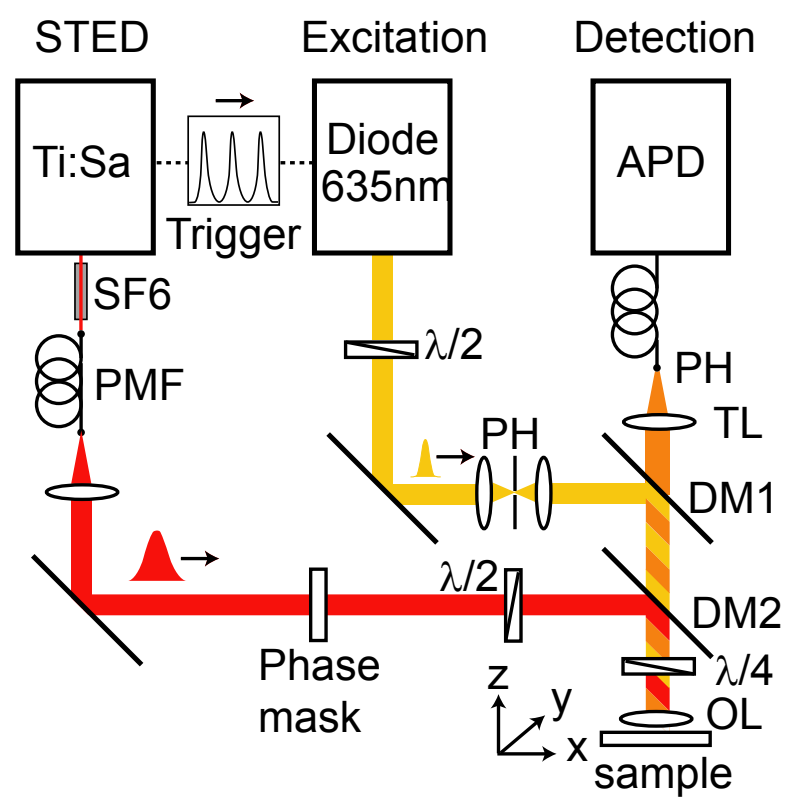

Figure 2.4: Experimental setup. For explanations about abbreviations see text.

On the outcoupling end, the beam is collimated by a lens before passing the phase mask that is used for the measurement. The STED beam is then reflected by the dichroic mirror (DM2) and focused into the sample. To overlay the excitation pulse with the STED pulse in time, the trigger signal generated by a photo diode located inside the STED laser, is the input signal of a custom-made delay unit which gives a time delayed output pulse. The delay of this pulse can be set with an accuracy of $10 \mathrm{ps}$. The delayed pulse sequence is utilized as the trigger input signal of the pulsed laser diode for the excitation. A $\lambda / 2$ retardation plate in the excitation and in the STED beam path, respectively, ensures in combination with the $\lambda / 4$ retardation plate in front of the objective lens the circular polarization of all beams.

For the image acquisition the position of the two beams is fixed, while the sample is moved with respect to the beam position. With this technique the setup is more compact and stable, although the scanning speed is limited by the stage.

\subsubsection{Resolution Measurements}

For the validation of the square root law from equation (2.7), the resolution of the STED microscope has to be measured with high accuracy. The most suitable sample would be a sparse distribution of stable point-like emitters. The problem of using single molecules as pointlike emitters is the weak fluorescence signal. Further problems of using single molecules are blinking of the dye and orientation effects as the cross section for stimulated emission varies with $\cos ^{2}(\phi), \phi$ defines the angle between the dipole orientation of the molecule and the polarization of the depletion beam [48][49]. Furthermore, the single molecule has to withstand the high resolution imaging without photobleaching or transitioning into a long living 


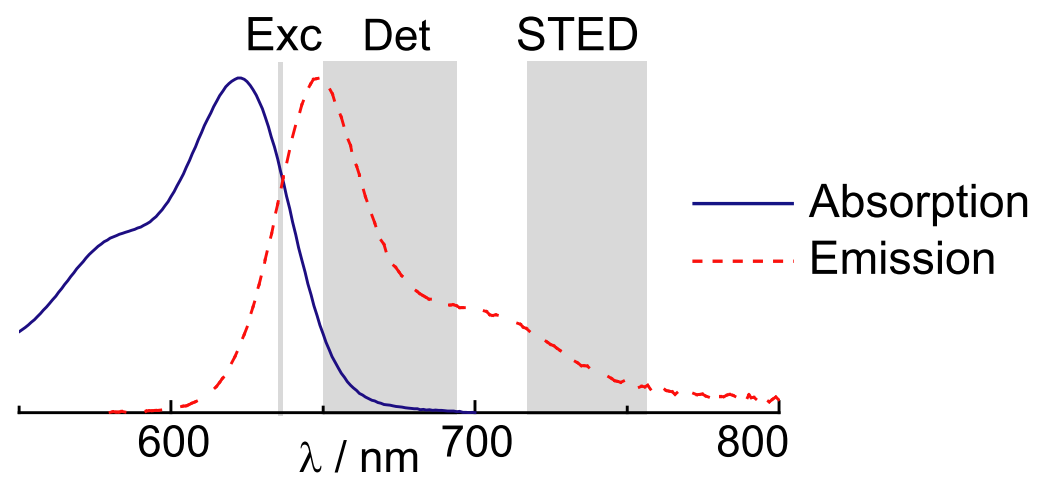

Figure 2.5: Absorption and emission spectrum of the crimson fluorescent spheres.

dark state. The alternative to using a point like emitter is to use a small object whose size is independently known from another measurement technique. The resulting image in the measurement is then the convolution of the effective PSF, whose size gives the resolution, with the object. When the size of the object is known and is smaller than the resolution of the microscope, the size of the effective PSF can be calculated (see appendix A.2.

For the presented measurements, the resolution was established by imaging fluorescent beads with a diameter of $24 \mathrm{~nm} \pm 4 \mathrm{~nm}$ (crimson fluorescent spheres, Invitrogen). The bead size was determined by the manufacturer with transmission electron microscopy. The spectrum of a bead solution is shown in figure 2.5. The advantage of this sample is the ensemble of dye molecules located inside the Polystyrol sphere. No orientation effects are expected and the fluorescence signal is large enough for a good signal to noise ratio. The STED wavelength is set to about $730 \mathrm{~nm}$ with the detection range for the fluorescence of $670 \mathrm{~nm} \pm 20 \mathrm{~nm}$.

The key phenomenon for the STED microscopy is the depletion of the fluorescent state of the dye. The depletion dependence on the STED intensity is presented in figure 2.6. For this measurement a diluted bead solution was spread over a Poly-L-Lysine coated cover slip before the beads were embedded in Mowiol A.5. The excitation and the STED beam without any phase mask are overlaid in space. A single bead is irradiated with a certain amount of excitation light (several $\mathrm{kW} / \mathrm{cm}^{2}$ ). The detected fluorescence was compared to the signal with the overlaid STED beam for different STED intensities (A.3). From this curve important parameters for the depletion process can be established. The saturation intensity can be immediately read off to $I_{S} \approx 7 \mathrm{MW} / \mathrm{cm}^{2}$. Another parameter is the remaining fluorescence for higher STED intensities, which is defined to be the level of the fluorescence that can not be depleted. A significant high level $(>15 \%)$ results in background signal in the STED image generated by the depletion pattern which ends in a weak signal to noise ratio. Reexcitation of the STED beam or, if working with linearly polarized depletion light, othogonal orientation of the molecule to the polarization of the STED beam could cause this remaining fluorescence. These crimson fluorescent spheres seem to be an adequate sample for STED microscopy because of the low saturation intensity with nearly no remaining fluorescence.

Due to the very high photostability, it was possible to measure the resolution increase 


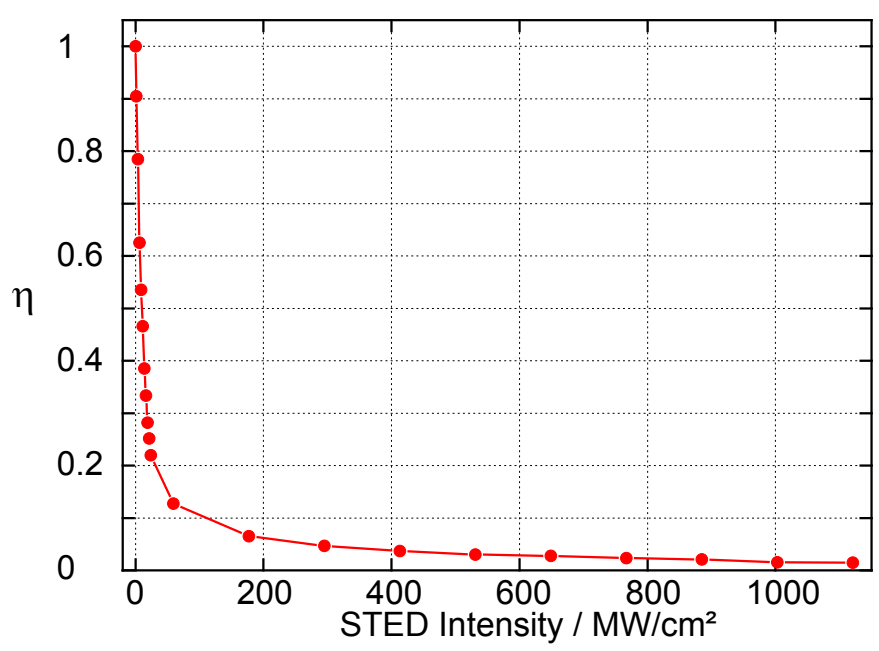

Figure 2.6: Depletion curve measured on single crimson fluorescent spheres fixed on a cover slip.

with increasing STED intensity on the same area of the sample. The measurements are shown in figure 2.7. The comparison of the confocal image (a) and the STED image at the highest possible STED intensity (b) shows the great benefit of a high resolution. The confocal data exhibits large objects with no resolved substructure. The STED image shows nearly every individual bead. Images were recorded with a pixel size of $15 \mathrm{~nm}$ and then interpolated to a pixel size of $10 \mathrm{~nm}$. This measurement was a part of a power series done on this sample. The STED intensity was increased image by image and the effect of this increase in intensity can be directly observed. The intensities of the STED beam used in the series were $0 \mathrm{MW} / \mathrm{cm}^{2}$ (confocal), $40 \mathrm{MW} / \mathrm{cm}^{2}, 158 \mathrm{MW} / \mathrm{cm}^{2}, 316 \mathrm{MW} / \mathrm{cm}^{2}$ and $1.7 \mathrm{GW} / \mathrm{cm}^{2}$. (c-g) present a magnification of the area marked by a white rectangle in (a) and (b). Starting with the confocal data (c), the intensity was increased to the maximum value (d-g). The corresponding depletion values can be read off directly from the depletion curve (h). Starting with an image with no resolved substructure, the real sample is transpired step by step.

For a quantitative investigation of the resolution of the STED microscope, a more diluted bead solution was used to prepare the sample. This sparser sample was then imaged with a pixel size of $10 \mathrm{~nm}$. A Gaussian fit to the intensity profile of a single bead gives the FWHM of the measured object size. The average of all presented single beads in the scan area, normally between 20 and 30, gives the mean FWHM with corresponding error bars. The resulting size of the effective PSF could then be calculated by the method explained in detail in the appendix A.2. Figure 2.8 presents the data of the resolution analysis. With increasing STED intensity (in units of $I_{S}$ ), the resolution capability of the microscope increases. The measured resolution values follows the fit to the square root function presented in chapter 2.2. The fitting parameters were $d_{c}=232 \mathrm{~nm}, a=3.48 \cdot 10^{-3} \mathrm{~nm}^{-1}$. These values are in very good agreement to the theoretical predicted values. The approximation of the shape of the depletion pattern in the lateral direction around the focal area by a parabola yields 

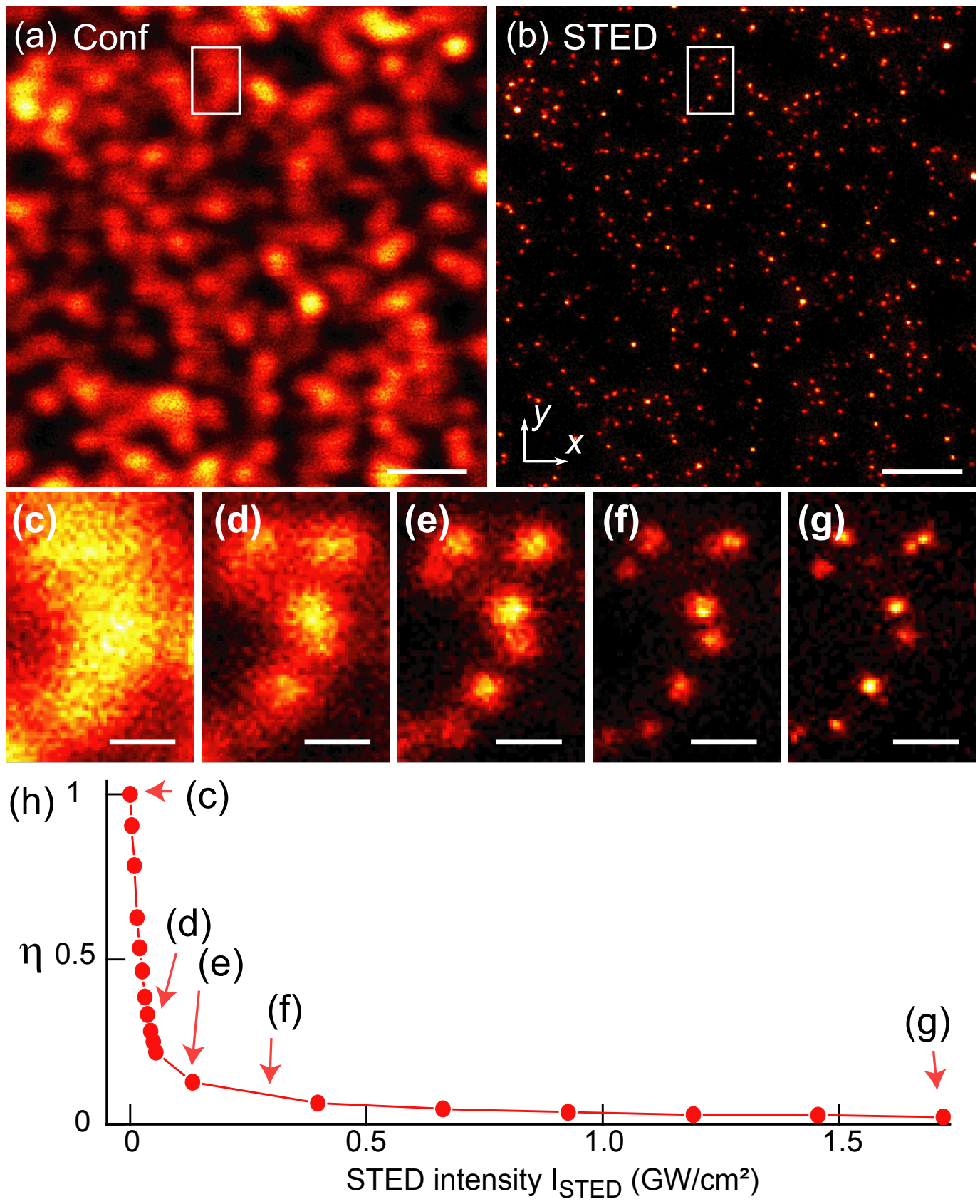

Figure 2.7: Resolution increase with increasing STED intensity. Confocal image (a) and the highest resolution STED image (b). The single power steps are highlighted in the insets (c-g) with the corresponding depletion value (h). Scale bars $1 \mu \mathrm{m}$, in insets $200 \mathrm{~nm}$ 


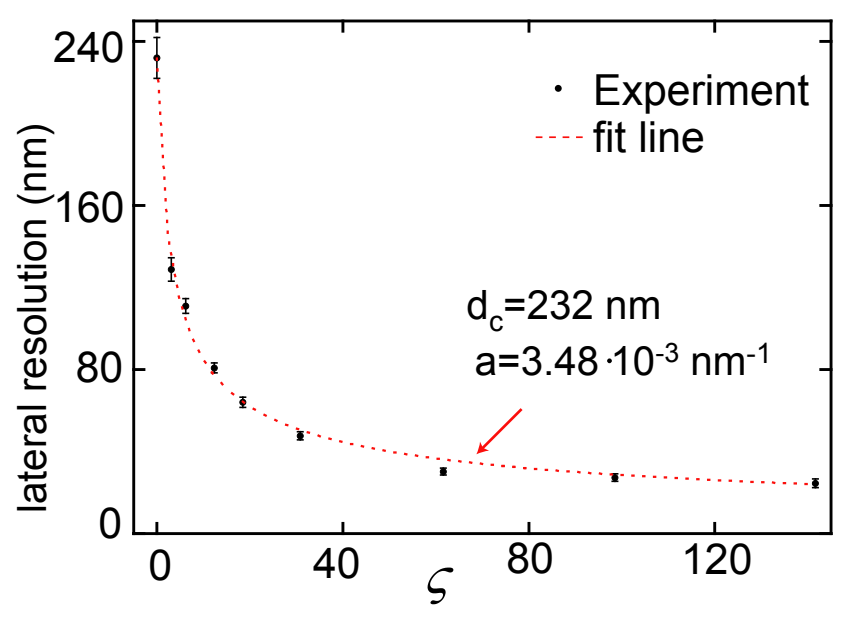

Figure 2.8: Quantitative analysis of the lateral resolution of a STED microscope. The points, which are measured values by averaging over the FWHM of several beads and calculate the effective PSF, are matching the fitted line for the square root law.

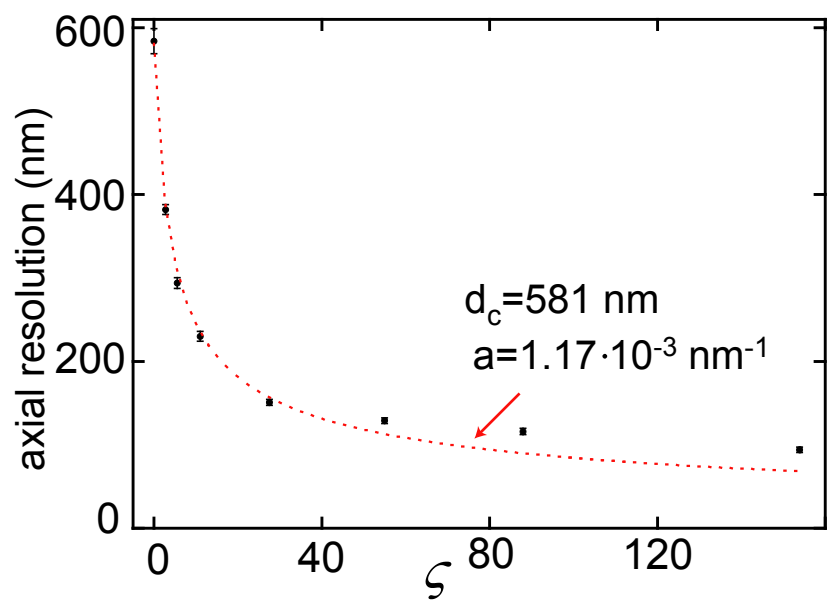

Figure 2.9: Axial resolution scaling in a STED microscope. Also for the depletion pattern, created by the phase mask $P_{3 D}$, the resolution follows a square root law (fit line).

excellent agreement in the theoretical and experimental data.

In the optimum, a resolution of about $25 \mathrm{~nm}$ was achieved in both lateral directions. This corresponds to a resolution enhancement factor of about 10 relative to the confocal mode.

For the analysis of the resolution scaling in the axial direction, samples of the same type were used. The helical phase plate $P_{2 D}$ was replaced with the axial phase plate $P_{3 D}$ to provide a confinement of the confocal PSF in the axial direction. Different $x z$-sections were recorded and the size of the beads were again determined by a Gaussian fit to the signal intensity profiles. Figure 2.9 presents the acquired data for the axial resolution. The fit of the square root law to the data gives the value for $a=1.17 \cdot 10^{-3} \mathrm{~nm}^{-1}$ and $d_{c}=581 \mathrm{~nm}$. The slight disagreement of the fit to the experimental values at higher saturation factors results 
from the fact that the beads are randomly distributed on the sample. This non-uniformity makes is difficult to ensure the perfect coincidence of the imaging plane with the object plane. For smaller sizes of the effective PSFs this effect comes into play. At any rate, the fitting parameters are again in a very good agreement with the theoretical predicted ones. The highest measured resolution is $94 \mathrm{~nm}$ which corresponds to an enhancement factor of 6 compared to the confocal mode.

\subsubsection{The Quality of the Minimum}

A very important parameter of the experimental STED setup is the quality of the minimum. A non perfect zero at the center of the depletion beam reduces the peak intensity collected from a small object. This peak reduction is very critical especially with relatively dark samples. It can limit the resolution of a STED microscope, because if the saturation factor reaches a certain limit, the fluorescence in the center of the focus will be reduced to a level where the signal to noise ratio is too weak to acquire an image [42]. A combination of a non perfect zero and a remaining - non depleted - fluorescence makes it even more critical as the resulting signal in the depletion pattern enhances the effective background level. As a result the signal to noise level is weaker.

As already mentioned, the generation of the doughnut shaped depletion pattern for the lateral directions was first done with a phase plate instead of a SLM. To estimate the residual intensity in the center of the depletion is a very meaningful test for this novel phase plate.

The measurements that were used for the resolution investigation in the lateral directions can also be used for the analysis of the minimum of the depletion pattern generated by the helical phase mask $P_{2 D}$. For this analysis, the peak intensities of isolated beads are compared for the STED and the confocal mode. To compensate for any bleaching effects that influences the result of this analysis, the confocal image was measured followed by the STED image of the same area. In a final step again a confocal measurement was performed. The peak intensity of an isolated bead in the STED image was then compared to the geometric mean of two confocal peak intensity values. For a statistical analysis this step was done for a large number of beads (20 to 30) and averaged.

With this method the peak reduction due to a depletion effect in the center of the STED PSF was analyzed. For this value of depletion the effective saturation factor $\varsigma_{z e r o}$ in the center of the PSF can be read out of the depletion curve measured on the same sample. The saturation factor $\varsigma$ at the outer rim (where the STED intensity is highest) of the PSF is known for every applied STED intensity from the depletion measurements as shown figure 2.6. Dividing $\varsigma_{\text {zero }}$ by $\varsigma$ gives the value $I_{z e r o}$ for the height of the minimum relative to the maximum intensity in the rim. These values are given in the table 2.2 .

The values of the table are illustrated in figure 2.10. The corresponding resolution enhancement factors relative to the confocal mode are displayed on the corresponding $x$ axis. For enhancement factors of about 4 , which is common in many biological applications, nearly no signal reduction could be observed. For a relative resolution enhancement of about 10 , the peak intensity drops down to about $65 \%$, which is sufficient for many practical applications requiring a high resolution. 


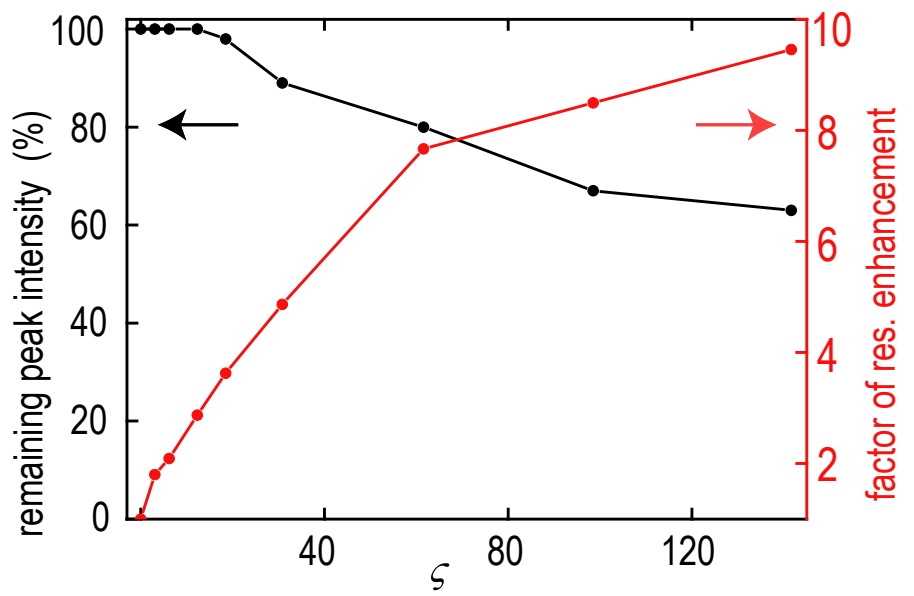

Figure 2.10: Remaining peak intensities with a high resolution STED microscope measured on single isolated beads. The corresponding resolution enhancement factor compared in red.

\begin{tabular}{c|c|c|c|c} 
STED intensity $/ \mathrm{MW} / \mathrm{cm}^{2}$ & $\varsigma$ & peak height $/ \%$ & $\varsigma_{\text {zero }}$ & $I_{\text {zero }} / \%$ \\
\hline \hline 0 & 0 & 100 & 0 & 0 \\
\hline 40 & 3.1 & 100 & 0 & 0 \\
\hline 80 & 6.2 & 100 & 0 & 0 \\
\hline 160 & 12.3 & 100 & 0 & 0 \\
\hline 240 & 18.5 & 98 & 0 & 0 \\
\hline 400 & 30.8 & 89 & 0.11 & 0.36 \\
\hline 800 & 61.6 & 80 & 0.3 & 0.49 \\
\hline 1280 & 98.5 & 67 & 0.72 & 0.73 \\
\hline 1840 & 141.6 & 63 & 0.8 & 0.56 \\
\hline
\end{tabular}

Table 2.2: Residual intensity $I_{z e r o}$ in the depletion pattern generated by the phase mask $P_{2 D}$ for different STED intensities.

\subsection{Summary}

In this chapter the quantitative analysis of the resolution scaling in a STED microscope has been shown. The equation for the resolution increase was derived in form of a modified Abbe equation, which gives the size of the effective focus - the resolution of the microscope - of the scanning spot. The modified Abbe equation exhibits no theoretical limit for the size of the effective focus whose size depends on three independent parameters: the saturation factor, the confocal resolution and a depletion shape dependent factor. The saturation factor depends only on the the saturation intensity, which is a dye characteristic parameter, and the depletion beam intensity. The confocal resolution is the parameter acting like a 'starting point' for the resolution. The shape dependent factor represents the curvature of the depletion pattern around the center of the focus. Within a very good approximation, the shape of the depletion beam can be written by a parabola. 
The approximation has been validated theoretically for two different depletion patterns the helical $P_{2 D}$ and the axial phase mask $P_{3 D}$. The PSFs of the single beams were calculated and the size of the effective focus acquired by considering the depletion mechanism. Out of the fitting process of the modified Abbe equation to the resolution data, the parameter for the confocal resolution and the shape depended constant for the two depletion pattern were established. The parameters provided by the experimental data for both depletion pattern are in very good agreement with the theory.

The residual intensity at the center of the depletion pattern is a very critical parameter regarding the resolution capability of the microscope. A residual intensity in the center of the depletion pattern drops the peak fluorescence in the STED image, which results in a weak signal to noise ratio. The realization of the depletion pattern itself was done for the first time by a phase plate, which results in a stable and compact setup. The quality of the minimum of the lateral depletion pattern, $P_{2 D}$, was analyzed by comparing the signal coming from the peak of an isolated fluorescent bead in the confocal image with that obtained from the corresponding STED image. The height of the residual intensity in the center of the depletion pattern calculated with this method was determined to about $0.5 \%$ according to a remaining signal height of about $63 \%$ for a resolution of $25 \mathrm{~nm}$ which corresponds to a resolution enhancement factor of about 10 compared to the confocal mode. With an enhancement factor of 4, nearly no signal reduction was observed. The resolution achieved in this chapter was not theoretically limited but due to practical constraints. The maximum STED power in combination with the cross section of the dye for stimulated emission gives the upper limit for the resolution. 


\section{3D Imaging - Resolution Enhancement in All Spatial Directions}

\subsection{Motivation}

Measurements with a 10-fold resolution enhancement relative to the confocal mode in two lateral dimensions have been presented in the previous chapter. The adoption of such an imaging platform is of the most utility in the examination of samples whose features of interest are restricted to two dimensions, and are not masked by variations in the remaining, third dimension. The bead measurements presented in section 2.3.2 constitute one such example, since a monolayer of beads was in contact with the cover slip, and a three dimensional structure was not expected. Some biological samples can also be prepared in such a manner. For example, an isolated cell membrane can be localized as a sheet on the surface of a cover slip. Utilization of this preparation technique allows two dimensional high resolution images of membrane proteins to be obtained, importantly, avoiding significant background signal [43][50][51]. These examples notwithstanding, the restriction to two dimensions is ultimately limiting. There are important samples where a high resolution in all three spatial dimensions is a prerequisite. The identification and mapping of morphology is a primary goal in the study of self organised three dimensional colloidal crystals. Furthermore, the 3D structure of the cell nucleus can only be explored when an enhanced resolution in the axial direction is achieved.

In order to place the challenge of '3D imaging' in context, it is important to recognize the distinction between 3D imaging and optical sectioning. '3D imaging' refers to the acquisition of a complete 3D data stack with the desired voxel size while optical sectioning involves obtaining a single slice or section with an enhanced resolution in three directions [45][39]. An important phenomenon encountered during the process of 3D imaging is that of photobleaching, which refers to the irreversible photochemical destruction of a fluorophore that is under illumination. Scanning a PSF with a small pixel size (relative to the size of the PSF) over the sample excites and deexcites dye molecules even when the latter are not in the central region of the PSF, where the fluorescence is being read out. The necessary reduction in pixel size frequently results in dye molecules getting bleached even before the detection of their fluorescence is attempted. Since this effect takes place in all spatial directions, a practical realization of 3D imaging must achieve both enhanced resolution in all directions, as well as negotiate the limitations placed by the onset of photobleaching. 
This chapter describes the experimental details of a system that achieves 3D imaging in densely packed colloidal nanostructures and biological samples.

\subsection{Experimental Results}

\subsubsection{Experimental Setup}

The experimental setup for 3D imaging is basically very similar to that described in chapter 2.3.1 and already described in this work [52]. The resolution enhancement in the axial direction is achieved by adding a second pathway for the STED beam that incorporates the phase mask $P_{3 D}$. The STED beam coming out of the single mode fiber is divided by a polarizing beam splitter (PBS) into two paths that possess orthogonal polarization, see figure 3.1 (a). One path incorporates the helical phase plate $\left(P_{2 D}\right)$, while the other, the axial phase plate $\left(P_{3 D}\right)$. After passing through the respective phase plates the two beams are recombined by a second polarizing beam splitter.

In the pulsed laser system employed, the coherence length is in the range of $\mu m$, which is less than the difference of the length of the optical paths. The recombination of the two beams thus results in an incoherent overlay. A $\lambda / 2$ retarder in front of the first beam splitter sets the relative intensities of the two STED paths.

Figure 3.1 (b) shows the measured PSFs of the individual beams as well as the overlaid PSF of the two STED beams. As a consequence of the polarization dependence inherent in the detection path used, it was not possible to measure the combination of $P_{2 D}$ and $P_{3 D}$ directly. However, the sum of the two PSFs clearly shows the potential for $3 \mathrm{D}$ resolution

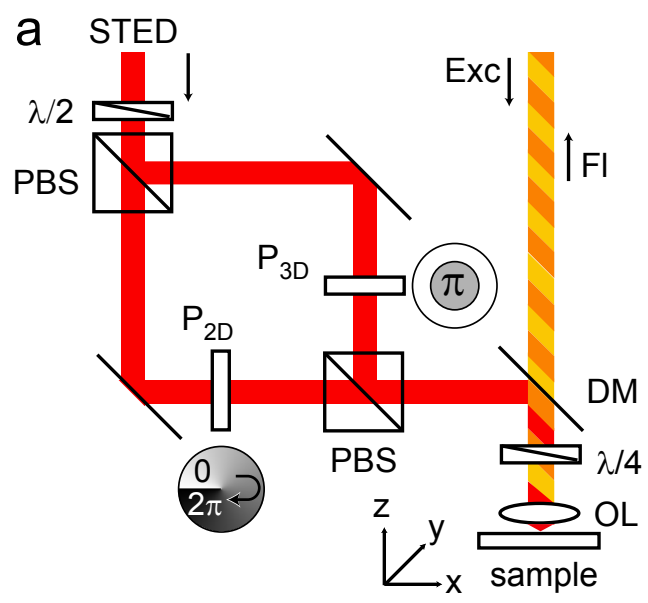

b

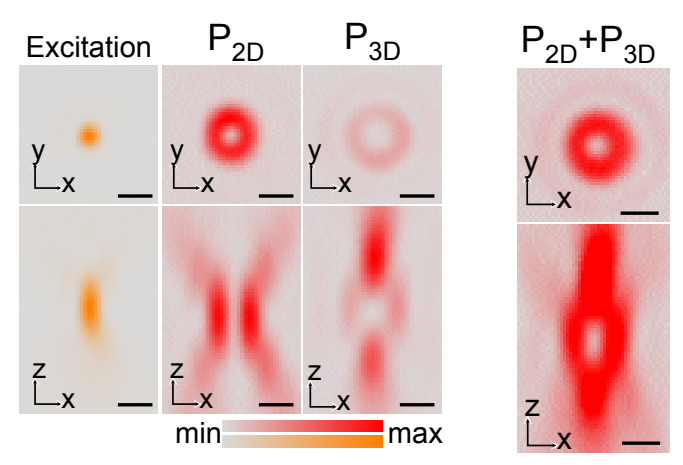

Figure 3.1: Experimental Setup used for 3D imaging (a). (b) The experimental PSFs for single beams as well as for the combined STED beams are presented. Scale bars $500 \mathrm{~nm}$. 
enhancement due to the reduction in the central focal region, i.e. in the region of zero STED intensity, in all three directions.

\subsubsection{The Focal Volume}

The focal volume conforms to the volume of excited molecules emitting a fluorescence signal. It can therefore be defined as the volume of the ellipsoid marked off by the FWHM in each spatial direction:

$$
V=\prod d_{i}
$$

where $d_{i}$ defines the FWHM in the particular direction $i=x, y, z$.
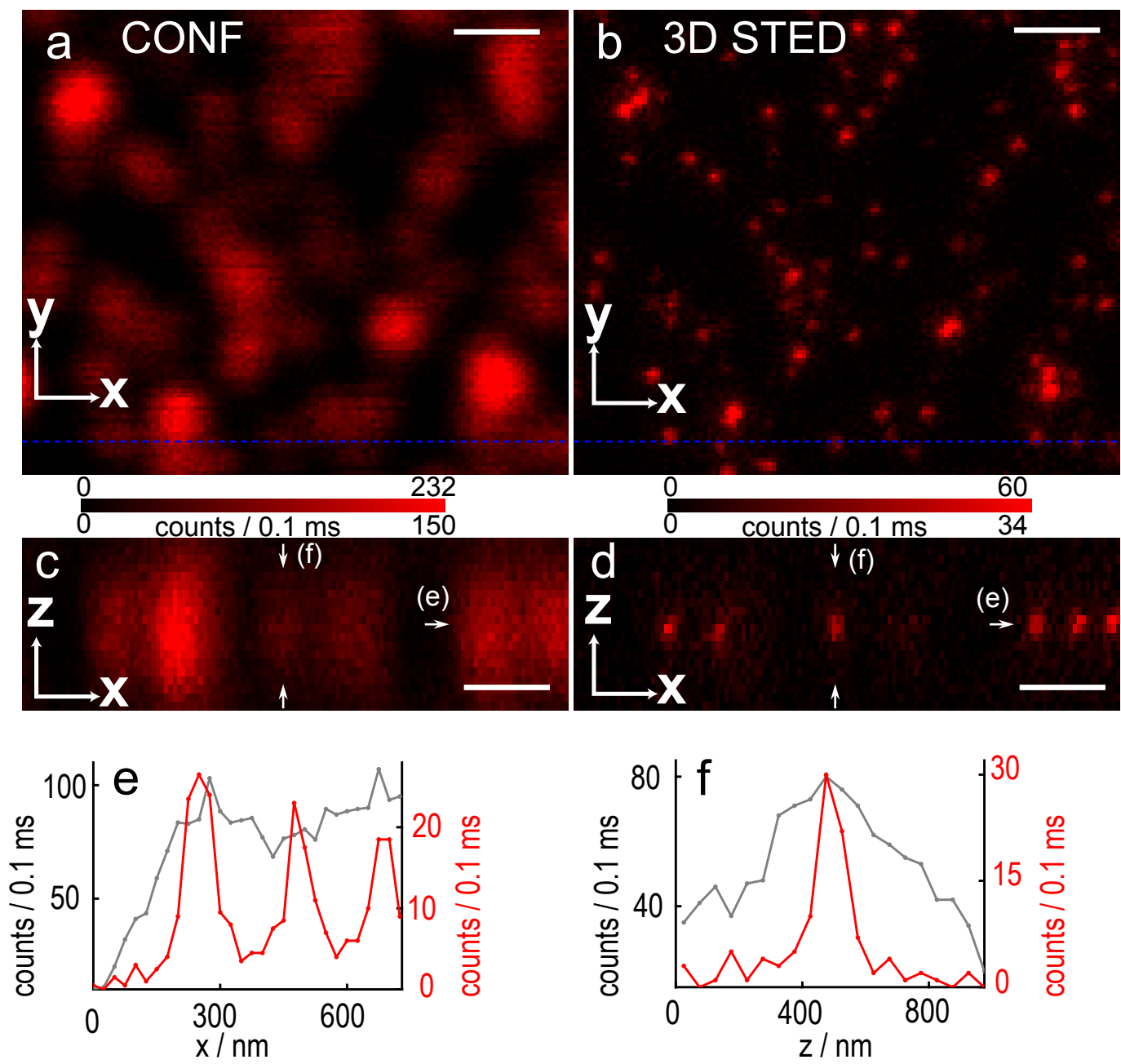

Figure 3.2: 3D data stacks of $24 \mathrm{~nm}$ fluorescent beads. (a,b) shows the confocal and STED image of an $x y$ section along the focal plane. An $x z$ section along the dashed blue line is presented in $(\mathrm{c}, \mathrm{d})$. Intensity profiles along the $\mathrm{x}(\mathrm{e})$ and $\mathrm{z}(\mathrm{f})$ direction indicated by white arrows in panel (c) and (d). Scale bars $1 \mu \mathrm{m}$. 


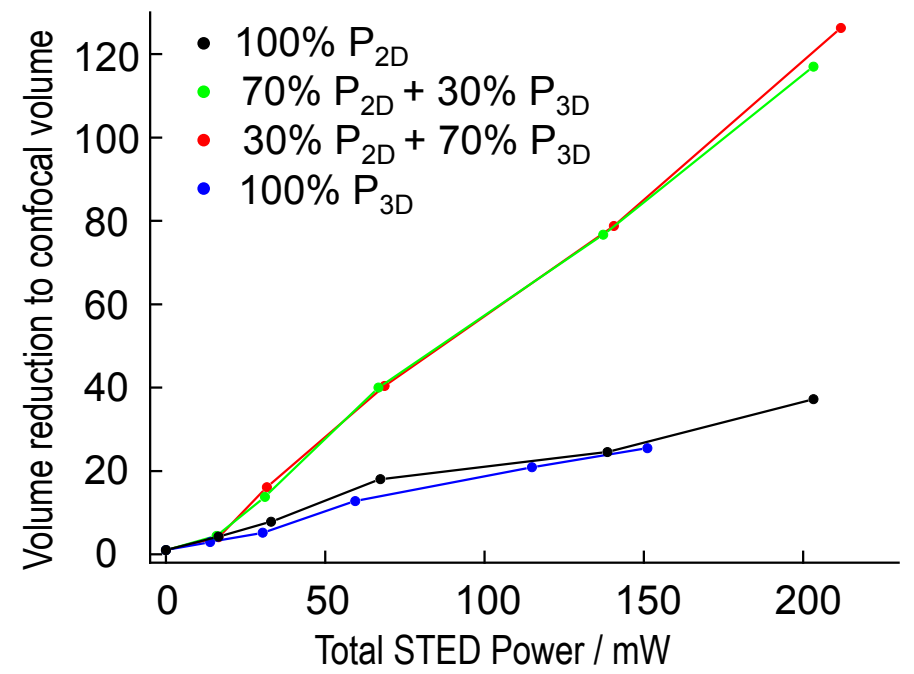

Figure 3.3: Reduction in focal volume obtained by combining the depletion patterns $P_{2 D}$ and $P_{3 D}$.

As already discussed in chapter 2 this volume can be reduced by quenching the excited molecules by using certain depletion patterns. The desired depletion pattern is obtained by placing a phase mask in the path of the depletion beam. The choice of the depletion pattern influences not only the focal volume, but also the shape of the effective focus [52]. The use of the helical phase mask, $P_{2 D}$, increases the resolution in the lateral plane dramatically, but leaves the axial direction untouched, which leads to a needle shaped focal volume. In case of the axial phase mask, $P_{3 D}$, the resolution is confined in all three directions, and the shape of the resulting focal volume is close to a sphere.

The optimum depletion pattern for any given sample is a function of the unique structural characteristics of that individual sample. In the case of a sample whose morphology is completely unknown and for which the structural features are required to be resolved in all directions, a spherical focal volume is recommended. The size of the sphere can be influenced by the intensity used in the STED beam. Once the size of this sphere is below the size of the features of interest in the sample, the quality of the acquired data is limited by the signal to noise ratio that is recorded. Thus, once the critical resolution is achieved, the signal to noise is optimized by further minimization of the focal volume.

The size of the effective PSF in each particular direction can be determined by measuring 3D data stacks of fluorescent beads with a diameter of $24 \mathrm{~nm}$ and performing an analysis in the manner explained in appendix A.2. A 3D stack measured using a combination of phase plates $\left(70 \% P_{3 D}+30 \% P_{2 D}\right)$ is shown in figure 3.2. Images (a) and (b) are the confocal and STED images of the lateral focal plane which clearly show the enhanced resolution in the lateral direction. An $x z$-section taken along the dashed blue line is shown to highlight the improved axial resolution with 3D STED (d) in comparison to confocal imaging (c). Intensity profiles along the lines indicated by the white arrows are displayed in (e) and (f). The presented raw data evidences resolution enhancement relative to the confocal imaging in all spatial directions. Using such measurements, the effective focal volume can 

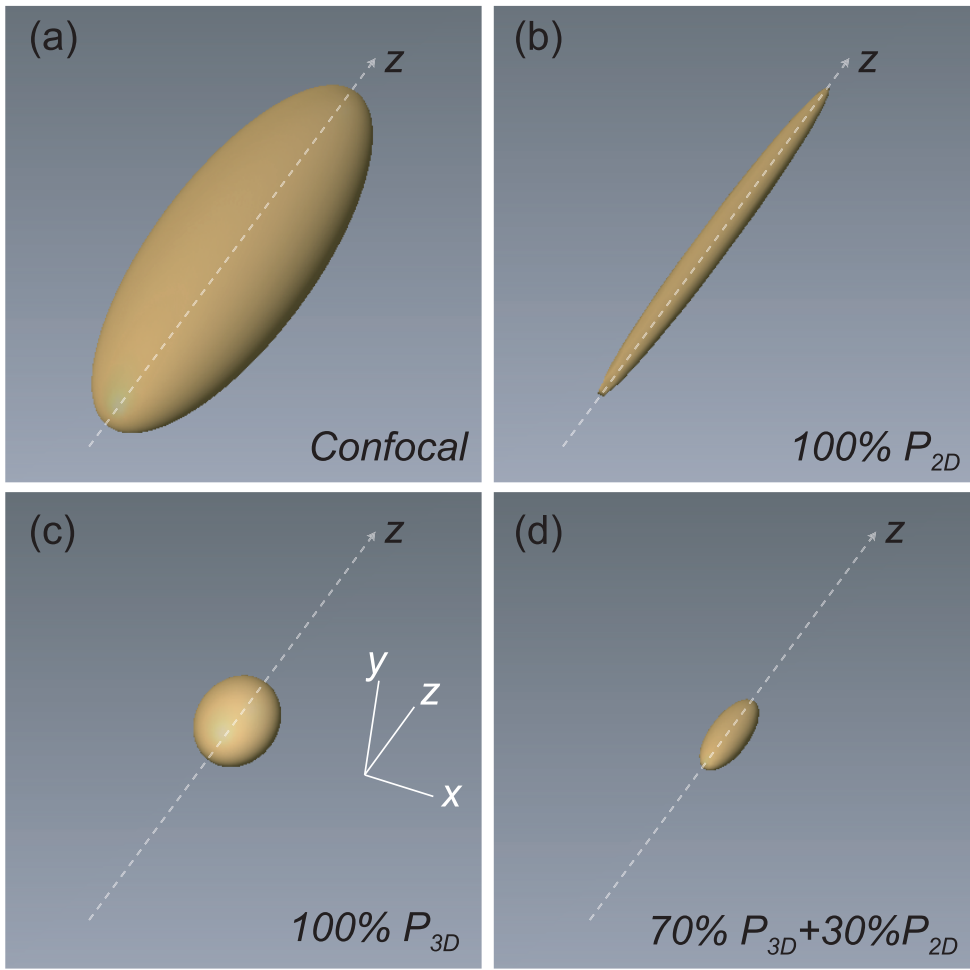

Figure 3.4: 3D illustration of the effective PSF while applying $150 \mathrm{~mW}$ STED power. Starting with the confocal PSF (upper left), the shape of the PSF changes for different depletion patterns.

be calculated for different power combinations of the two depletion patterns. The reduction of the focal volume relative to the confocal focal volume is plotted in figure 3.3 . The $x$ axis depicts the total power of the STED beams (i.e. the addition of the two single beams) in the focus. The incidence of photobleaching is an important consideration in the acquisition of 3D data stacks. Maintaining the same total STED power should result in a similar amount of bleaching. Assuming the critical resolution has been achieved, it can clearly be seen from the graph that for the same amount of bleaching, the combination of phase plates results in a better signal to noise ratio. Note that a slightly lower maximal power was applied in the case of the single pattern due to practical limitations in the setup, which was used for both single and combination measurements. From the graphs it can be seen that the single phase masks, $100 \% P_{2 D}$ or $100 \% P_{3 D}$, achieve a volume reduction factor of about 20 relative to the confocal mode, while the combination of the two phase plates $\left(70 \% P_{3 D}+30 \% P_{2 D}\right.$ or vice versa) gives an enhanced reduction factor up to 125 . In absolute numbers, the confocal focal volume is about $162 a \mathrm{~L}$, while the best focal volume achieved with the combination of two depletion patterns is $1.3 \mathrm{aL}$.

To get an idea of how the shape of the focal volume looks for different depletion patterns, figure 3.4 shows a three dimensional view on the effective PSFs. For the figure, the PSFs were approximated by a 3D Gaussian with corresponding measured FWHM in the particular directions. Starting with the confocal PSF (a) the shapes of the effective PSFs with a con- 
stant applied STED power of $150 \mathrm{~mW}$ can be compared. Applying only the helical phase mask $P_{2 D}$ yields a needle shaped PSF (b), because no confinement in the axial direction is provided. A nearly spherical focal volume can be achieved by applying the axial phase plate $P_{3 D}$ to the depletion beam (c). The combination of the two phase plates leads to the smallest focal volume, which can be clearly observed in the surface rendered view (d).

As a result, the presented 3D measurements shows a resolution of $43 \mathrm{~nm}$ in the lateral direction and $125 \mathrm{~nm}$ in the axial direction within a 3D data stack by implementing the combination of two depletion PSFs. These measurements demonstrate the ability of doing 3D imaging on the nanoscale using STED microscopy.

\subsubsection{Three-Dimensional Nanoscopy of Colloidal Crystals}

Colloidal materials are dispersions in which the suspended items are much larger than the other components in the system. The ability afforded by colloidal systems to easily tune a number of variables including the forces of interaction, size, shape and composition has led to their popularity as model systems for atoms and molecules. They have thus been employed in the study of a variety of phenomena in condensed matter physics including crystal nucleation and growth [53], phase transitions such as melting [54], and the glass transition [55]. In addition, the capability of colloids to self organise into crystal structures has resulted in their application as advanced materials such as photonic crystals [56]. Imaging two dimensional colloidal crystals is easily realized by electron microscopy [57][58]. However, in the bulk of the cases, the 3D morphology of the colloids is of primary interest. Confocal microscopy has thus emerged as the most popular tool for the 3D mapping of colloidal crystals [59]. However, the use of confocal microscopy with its associated limitation in resolution, especially in the axial direction, restricts the size of the particles to approximately the wavelength of the light being employed. For noninteracting, "hard sphere", colloids the size of colloidal particles affects the sedimentation and overall dynamics. While for particles with more complex interactions and shapes, the size and orientation of the particles also affect the relative magnitudes of the attractive and repulsive forces that govern the process of self-assembly.

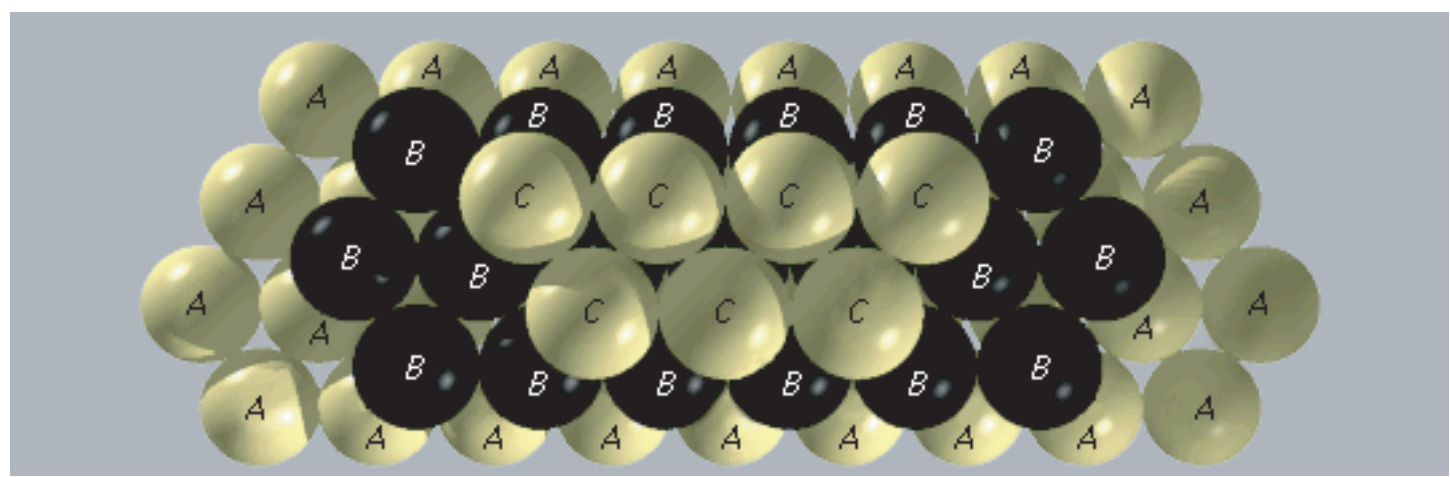

Figure 3.5: Close packed spheres are organized in self organized layers (A,B and C). (C)Encyclopaedia Britannica, Inc 


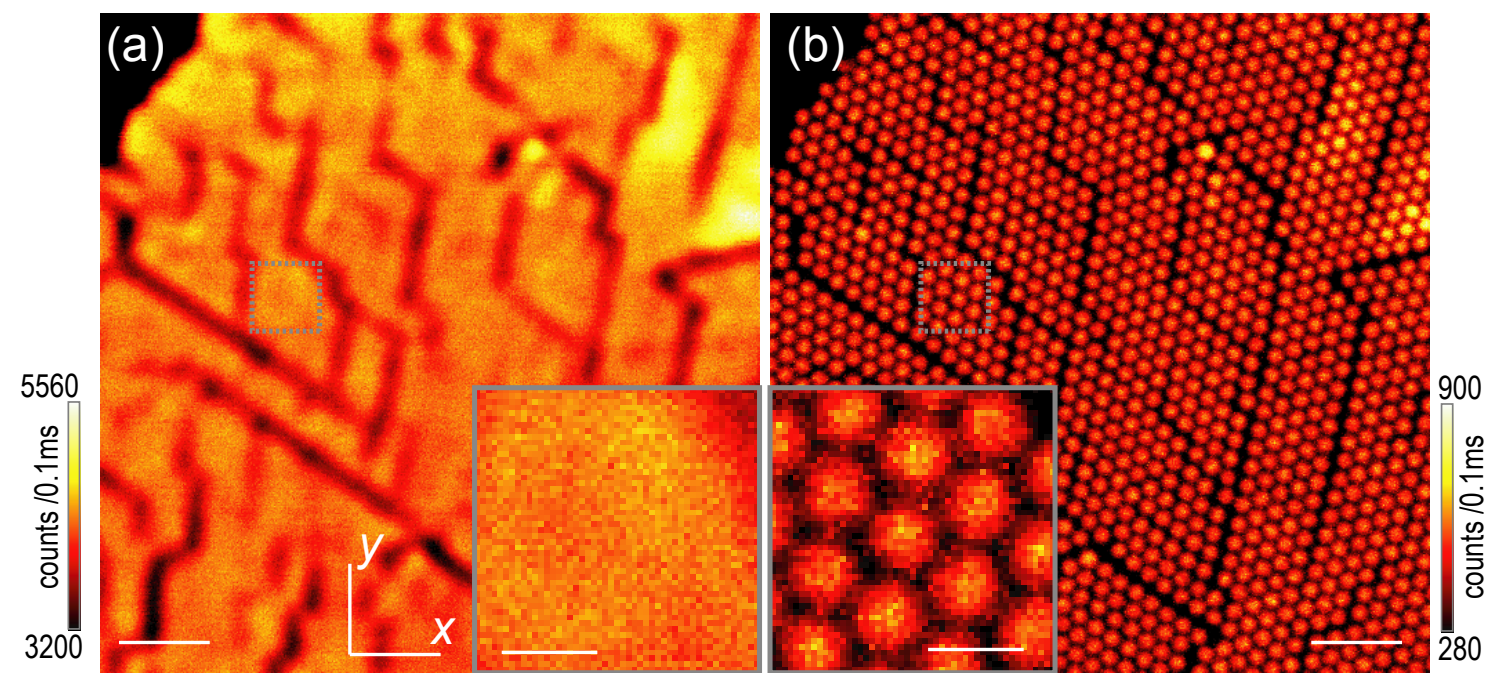

Figure 3.6: Measurement of 1 layer of hexagonal close packed $200 \mathrm{~nm}$ spheres. Left the confocal, right the STED image. Raw data. Scale bar $1 \mu \mathrm{m}$, in the insets $250 \mathrm{~nm}$.

Thus imaging with an enhanced resolution in 3D would have strong implications for the study of colloidal systems From an imaging perspective the most effective demonstration of the 3D imaging of colloidal crystals would be the mapping of densely packed particles. For particles of a single size, close packed translationally periodic structures can be either face centered cubic or hexagonal close packed. In order to understand the structure of these crystals it is convenient to view them as single layers of hexagonally close packed spheres stacked in sequence. The registration between subsequent close-packed layers determines whether the resultant crystal structure is face-centered cubic (ABCABC... sequence, fcc for , fcc for short), hexagonal close packed (ABABA... sequence, hcp) or random hexagonal close packed (r-hcp). Figure 3.5 is a schematic of the packing sequence of spheres arranged on a face centered cubic lattice.

The different layers $\mathrm{A}, \mathrm{B}$ and $\mathrm{C}$ are shifted with respect to one another, such that the center of the spheres in the second layer (B) are positioned in the holes of the first layer (A). In the third layer $(\mathrm{C})$, the centers of these spheres are positioned above the remaining holes of the first layer. Adding a fourth layer to the system repeats the first layer.

To demonstrate the ability to map the morphology of 3D colloidal crystal structures, crystals consisting of fluorescent spheres with a diameter of $200 \mathrm{~nm}$ were used ${ }^{1}$. The crystals were organized by a technique known as convective assembly, which involves the selforganization of spheres at the moving contact line created by the controlled evaporation of a solvent [58][60][61]. A substrate is withdrawn from a solution containing the fluorescent beads at a fixed rate. Particles drawn into the meniscus are deposited onto the surface of the moving substrate. The structure and thickness of the sample being deposited is a function, among other factors, of the concentration and size of the particles in the solution, the evaporation rate of the solvent, and the withdrawal rate of the substrate.

\footnotetext{
${ }^{1}$ Samples prepared by C. K. Ullal, Department of Nanobiophotonics, Göttingen
} 


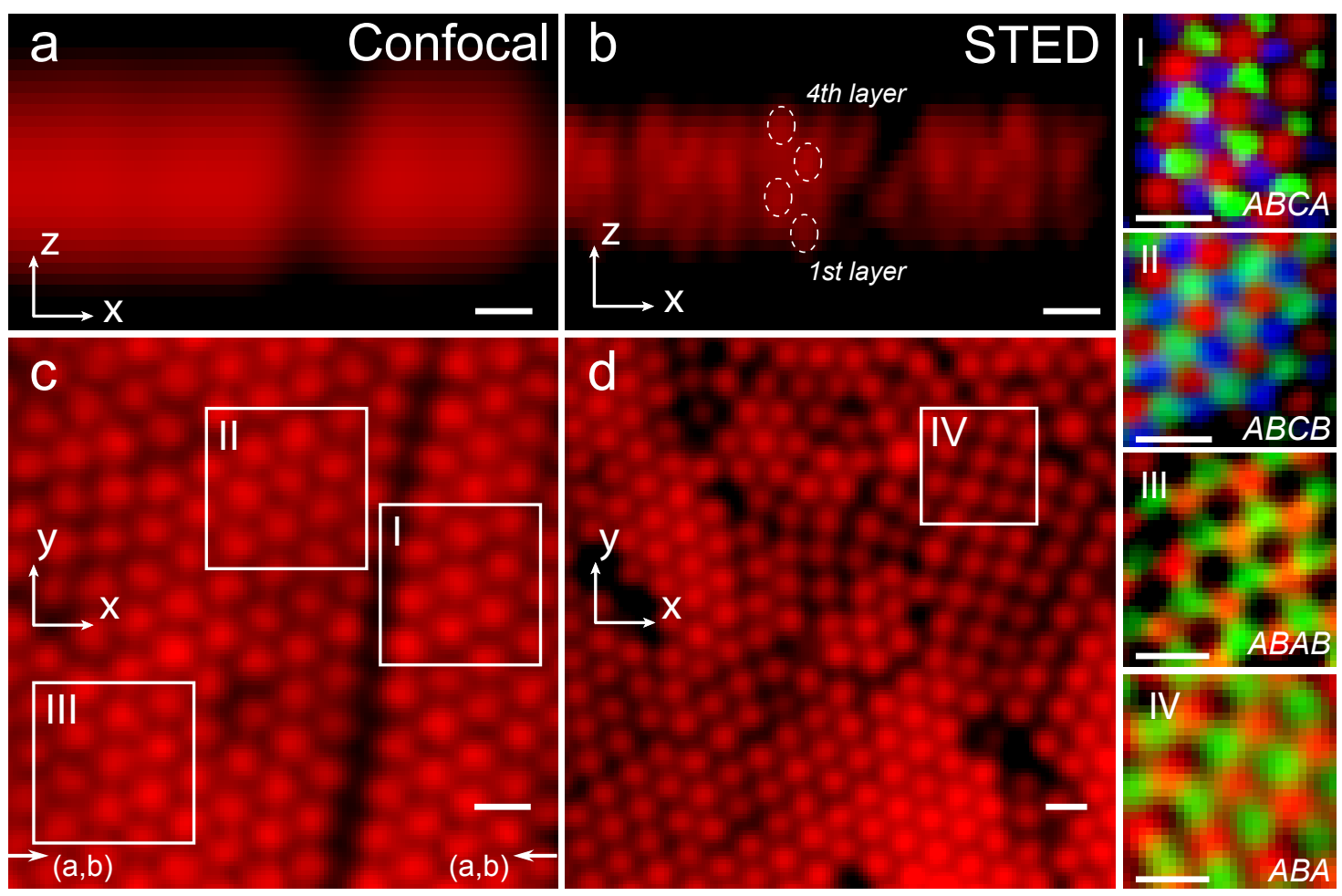

Figure 3.7: 3D nanoscopy of convectively assembled 3D colloidal crystals. (a,b) $x z$ section of 3D data stack from confocal (a) and STED (b) image of a four layer crystal. The $x y$ section of the first layer is shown in (c). (d) presents the first layer of a 3-layer crystal. The right column shows projection images (1st layer red, 2nd blue, 3rd green) of the insets in (c) and (d). Scale bars $250 \mathrm{~nm}$.

Samples fabricated under varying conditions were examined.

Figure 3.6 shows an example of a one layer crystal imaged with an improved resolution in the lateral directions. In the confocal mode (a) just the defect lines of the sample can be observed, while even the raw data of the corresponding STED measurement (b) allows the identification of each individual bead. This measurement shows the improvement using STED microscopy for studying colloidal particles. Even a single layer in the lateral plane can not be resolved in the confocal mode while the STED technique easily resolves the structure.

Investigating crystals consisting of more than one layer requires an improved resolution in all directions realized by the combination of the two mentioned phase plates (70\% $\left.P_{3 D}+30 \% P_{2 D}\right)$. Representative, 3D linearly deconvolved images of some of the 3D structures examined are shown in figure 3.7. (a) and (b) clearly demonstrate the benefit of the 3D STED microscope. No structural features can be observed in the confocal $x z$-section while the STED image reveals the single layers of the observed crystal. A STED $x y$-section along the first layer of this crystal is shown in (c). The projection of the different layers of the marked areas (I-III) are highlighted in the right column. The different layers are overlaid after being color coded. The 1st layer is colored red, the 2 nd blue and the 3rd layer green. 


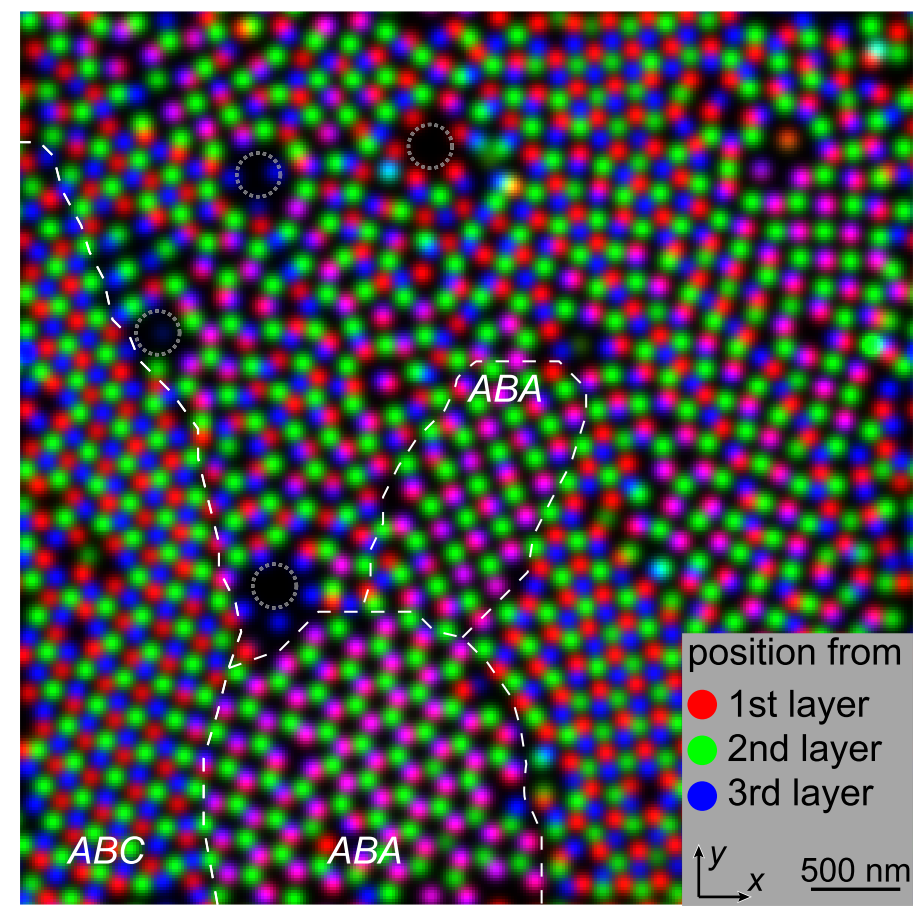

Figure 3.8: Measurement of a 3 layer crystal. After localization of the position of the spheres, a projection of the layers shows the packing sequence of the crystal.

Mixed colors result from the overlay of two layers with identical $x y$ registration. Separate regions displaying fcc, $\mathrm{r}$-hcp and hcp structures can be identified in this measurement. A three layer crystal corresponding to slightly different preparation conditions is shown in (d). The projection image of the marked inset (IV) clearly reveals the presence of a body centered cubic crystal.

The morphology of the crystals being examined can be further clarified by employing an algorithm to localize individual spheres 2 . The image stacks were deconvolved with a $150 \mathrm{~nm}$ diameter 3D Gaussian function which resulted in enhanced contrast and elimination of noise. By marking each pixel that displays a local maximum in intensity within an area of interest, it was possible to identify each sphere. However, the influence of noise and the presence of oversized spheres resulted in the marking of some positions $(1-3 \%)$ that did not correspond to a sphere in the raw data. These positions were removed manually. Finally, the positions of the centers of the spheres were found by calculating the local center of mass around each marked pixel with a precision of $50 \mathrm{~nm}-80 \mathrm{~nm}$. The resulting grid of points was then convolved with a sphere of $100 \mathrm{~nm}$ diameter. An example of this procedure is shown for the measurement corresponding to figure 3.7 (d). The projection image of the complete measurement, colorized as per the sequence specified earlier, is presented in figure 3.8

\footnotetext{
${ }^{2}$ Jan Keller, Department of Nanobiophotonics, Göttingen
} 

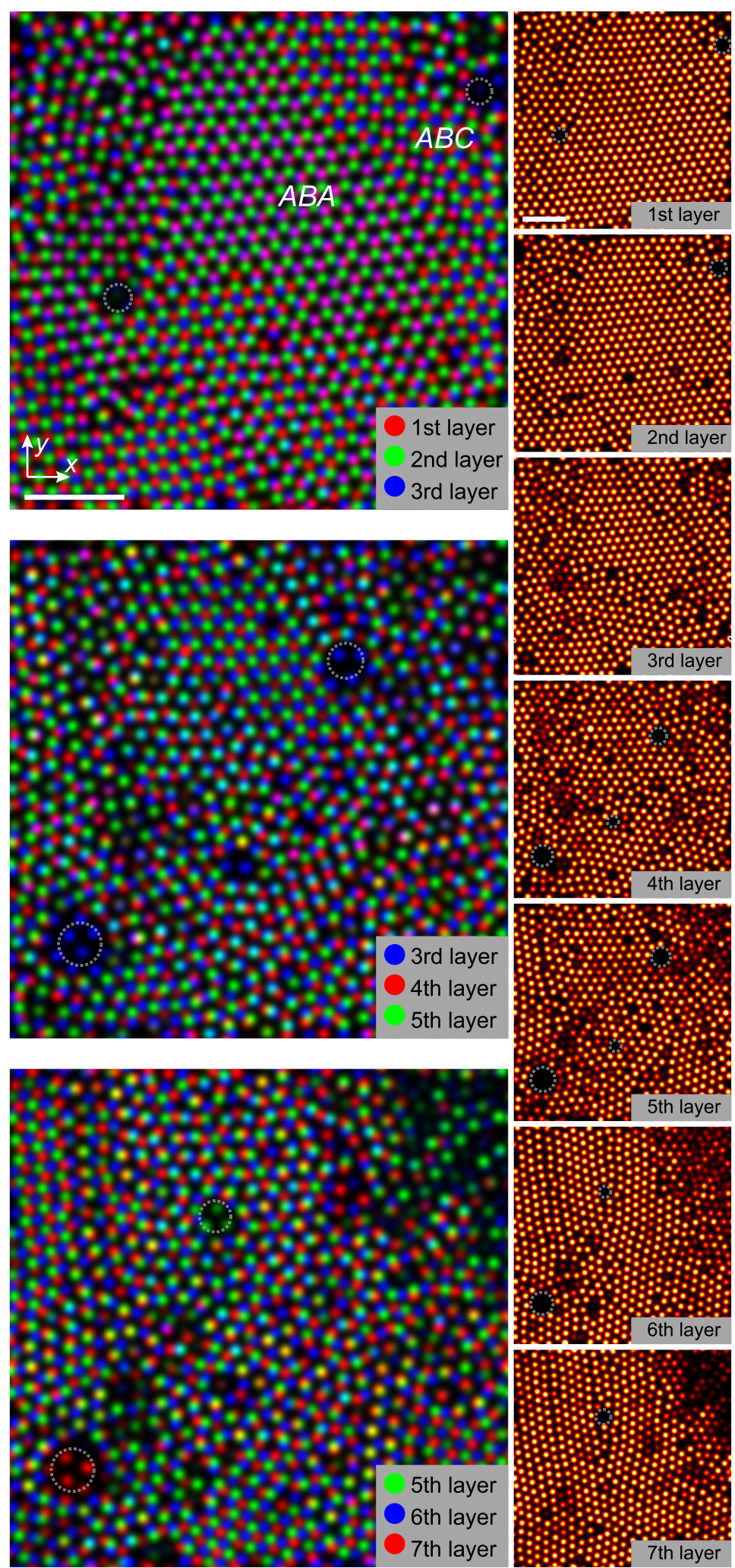

Figure 3.9: Reconstruction of a measurement of a 7 layer crystal. Scale bar $1 \mu m$. 
Manually removed artifacts, like larger spheres, are marked with a gray dotted circle. The different crystal structures are clearly evident in this area of the sample. The registration sequence of the face centered cubic lattice results in regions that contain all three colors. Areas made up by the hexagonal closed packed structure can be identified by their purple color which results from the overlay of the first layer (red) and the 3rd layer (blue). A body centered cubic (ABAB with square packing) can be observed in the center of the image.

By backfilling the samples with an immersion oil, it was possible to image thicker crystals. This prevents the loss of information associated with beam abberations that are typically observed in scattering media. Figure 3.9 shows a measurement of a 7 layer crystal. Once again the localization algorithm was used to clarify the crystal structure.

3 layer sets corresponding to the stacking sequence of the face centered cubic as well as hexagonal close packed crystal structures are seen. Over the full 7 layers, however, no preferential packing structure can be assigned.

\subsubsection{Three-Dimensional Measurements in Biological Samples}

The benefits of resolution enhancement in all three directions is not limited to colloidal crystals. In this section we demonstrate the feasibility of employing the experimental platform in the imaging of biological samples.

The sample used for testing the ability of 3D imaging is the nuclear lamina, where the meshwork, constituted by filaments of Lamin proteins, adds mechanical support within the nucleus. These intermediate filaments are classified in Lamin A, B and C. Lamin A and $\mathrm{C}$ are comprised of a set of three proteins arising from the same gene, while Lamin B is made up of two proteins arising from a different set of genes. These proteins (in this case Lamin A and C) can be immunolabeled with the antibody conjugated dye Atto 647N (AttoTec, Germany). This is achieved by specifically binding a primary antibody to this protein. Subsequently, secondary antibodies decorated with dye molecules are bound to the primary antibody, thereby providing the desired staining ${ }^{3}$

For the measurement of the nuclear lamina, the use of the depletion pattern generated by the axial phase plate $P_{3 D}$ is sufficient, as no small structural features are expected in the lateral directions. Figure 3.10 presents a 3D data stack of the cell nucleus. The measurement was performed with a water objective lens (63x NA 1.20, HCX PL APO, Leica Microsystems, Germany). Water also acted as the embedding medium for the cell. In order to reduce the incidence of bleaching, a triplet quenching buffer solution ${ }^{4}$ was mixed into the water. Its main effect is an effective reduction of the oxygen in the solution via glucose oxidation. The data stack shows the benefit of 3D STED. The surface rendered view shows no structure in the confocal mode (a), while the STED image identifies the nuclear lamina as an empty nucleus (b), which conforms with the expected structure of the nuclear lamina A and C. Raw data of single $x z$-slices directly evidences the resolution enhancement in the STED image (d) relative to the confocal one (c).

\footnotetext{
${ }^{3}$ Samples prepared by R. Medda, Department of Nanobiophotonics, Göttingen

${ }^{4}$ Provided by Robert Kasper, Applied Laser Physics and Laser Spectroscopy, University of Bielefeld
} 

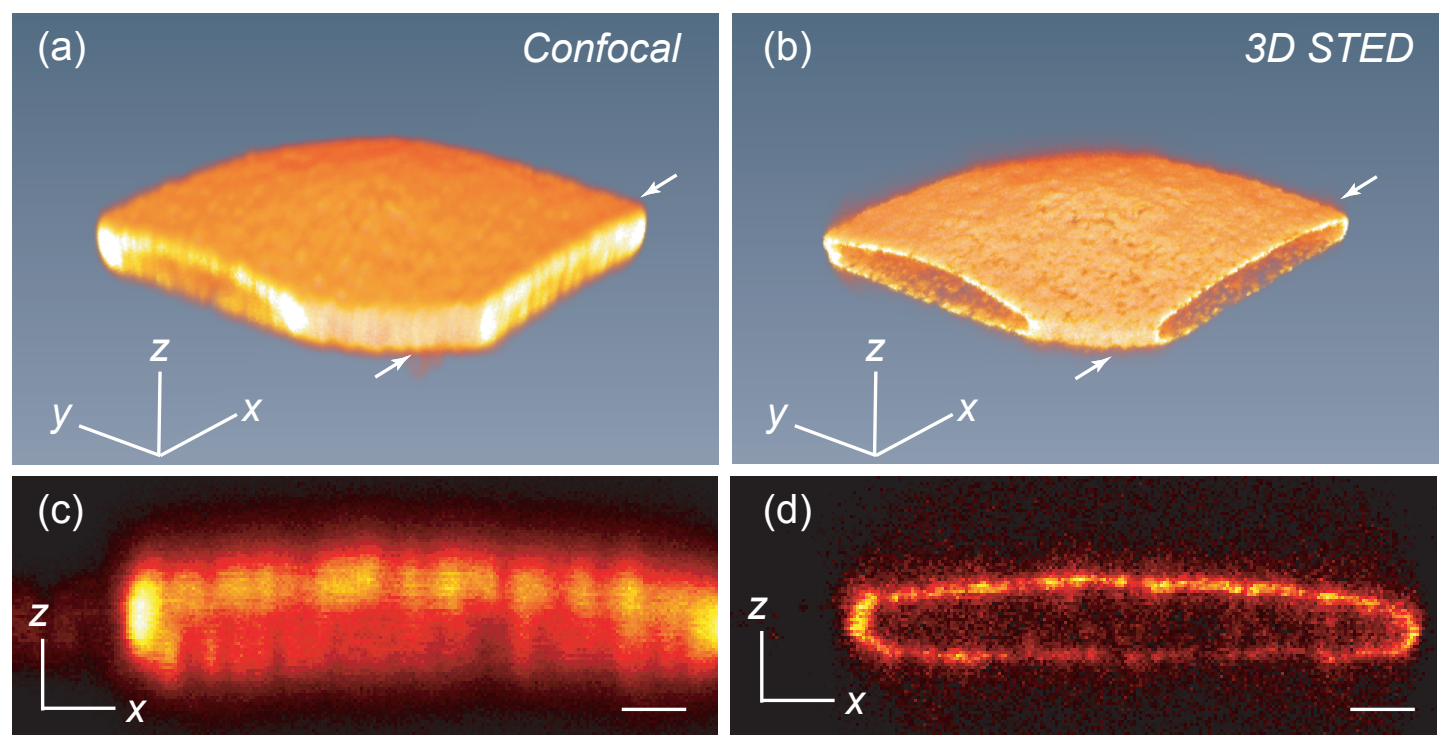

Figure 3.10: 3D data stack of the cell nucleus. The proteins Lamin A and $\mathrm{C}$ were immunolabeled with the dye Atto 647N. Scale bar $1 \mu m$.

\subsection{Summary}

In this chapter the capability of STED microscopy to perform 3D high resolution imaging has been presented. The investigation of the best depletion pattern showed the need for the introduction of a combination of two different phase masks in the path of the depletion beam. The use of the helical phase plate $P_{2 D}$ as the only phase mask ends in a needle shaped effective focal volume, which is not convenient for the investigation of $3 \mathrm{D}$ samples. The axial phase plate $P_{3 D}$ generates a depletion pattern which results in an isotropic shape of the effective focal volume. This depletion pattern enables the possibility of 3D imaging in unknown biological and technical samples. However, it has been shown that once a critical resolution is achieved, at which all structures of interest can be resolved, the focal volume can be further reduced by adding a second beam with the helical phase mask. A reduction in the focal volume always leads to an enhanced signal to noise ratio. With the incoherent overlay of these two depletion beams, an effective focal volume that was 125 times smaller than the corresponding confocal one, was demonstrated.

This optimized experimental setup was used to examine densely packed fluorescent bead nanostructures, thereby showing the huge benefit of 3D STED. The crystal structure formed by the convective assembly of $200 \mathrm{~nm}$ spheres was analyzed and allowed the identification of face centered cubic, hexagonal close packed and body centered cubic structures. Measurements of up to 7 layers were performed without any significant signal reduction. This work has strong implications not just for the colloidal sciences but also for the broader area of the materials science of nanostructures.

The measurement of a cell nucleus showed the feasibility of performing 3D imaging 
in biological tissue. The 3D data stack which was recorded without the onset of excessive photobleaching showed the structure of the nuclear lamina, which is located in the nuclear membrane. As the mechanism of reforming the cell nucleus after mitosis [62] has not been completely analyzed, imaging with an enhanced resolution will offer new information. This lays the groundwork for applications in the field of biological 3D imaging. 


\section{STED Microscopy with Continuous Wave Lasers}

STED microscopy with pulsed laser systems has been described in detail in the previous chapters. In this chapter the possibility of using continuous wave $(\mathrm{CW})$ lasers for high resolution microscopy is discussed. Following the introductory part, a theory of the depletion process with the modified experimental setup will be shown. Then measurements in technical and biological samples, in the lateral as well as in the axial direction, are presented.

\subsection{Simplify the Setup with CW - Motivation}

All the presented measurements up to now were recorded by a setup using pulsed lasers for excitation and STED. The realization of a pulsed STED system requires a more complex optical setup and additional electronic equipment: The pulse length has to be adapted to the requirements of the system (changing $f s$-pulses to a few hundred $p s$ ). The excitation and STED pulses have to be aligned in time, which requires complex electronics. Using CW lasers would simplify the setup, which also results in a price reduction of the system. The expense of CW laser sources are much lower than those for the pulsed ones. The expensive pulsed laser diode for the excitation light can be replaced by a very simple device - in principle by a conventional laser pointer - due to the low power requirements. The availability of CW laser sources, in particular for the excitation, is much wider than that for the pulsed ones, especially regarding the choice of the wavelength. For pulsed laser systems the most common laser is a Titanium:Sapphire laser whose wavelength is limited to the near-infrared regime. Considering the availability of many different dyes in the visible wavelength regime, a shift to this regime is desired. But this shift requires a second additional laser system like an optic parametric oscillator (OPO), which makes a pulsed STED setup in the visible range very complex and expensive.

Another important benefit in CW microscopy is that one is working with low peak intensities, since photodamage mechanisms scale with at least the square of the intensity of the irradiated light [63]. This fact has to be considered when imaging living cells, where any kind of photodamage induced in the cell changes its behavior and the biological evidence.

One advantage of a pulsed system is that high intensities can be achieved easily by a standard laser, in this work a Titanium-Sapphire laser. High intensities result in a high depletion efficiency - and therefore a high resolution (see chapter 2.2). The depletion efficiency should remain at its level, if the pulse length of the STED beam (in the applied setup $300 \mathrm{ps}$ ) with the same average power is stretched to the length of the fluorescence life time of the 
depleted dye, which is typically in the range of several $n s$. However, as the pulse separation of a $76 \mathrm{MHz}$ system is about $13 \mathrm{~ns}$ and therefore larger than the typical fluorescence life time, a higher CW laser intensity becomes necessary in comparison to the displayed system. Also, due to the constant flux of the depletion photons, a CW laser source for the excitation beam should be used. A theoretical approach to the relation between the relative powers required in pulsed and in $\mathrm{CW}$ mode follows in the next section.

\subsection{A Theoretical Approach to CW STED}

To compare the efficiency of the depletion process in the $\mathrm{CW}$ mode with the one in the pulsed system, the rate equations of the system have to be taken into account. The main assumption is that only two levels, the ground state $S_{0}$ with the population $N_{0}$ and excited state $S_{1}$ with the population $N_{1}$, contribute to the photo-physical system. The corresponding system is the same as already described in section 1.2.2 (figure 1.1). All the transition rates are the same, because the efficiency is compared in principle for the same molecule. The theoretical approach to solve this system is different in the CW mode than in the pulsed mode [34]. A time separation of the excitation and the STED transition is not justifiable for the case of CW excitation and $\mathrm{CW}$ depletion. Therefore the probability $k_{\text {exc }}$ for the transition of the ground state $N_{0}$ to the first excited state $N_{1}$ has to be taken into account. The excited state can either relax to the ground state by emitting a fluorescence photon with the probability $k_{f l}$ or by deexcitation in form of stimulated emission with the rate $k_{S T E D}$.

Two assumptions are made to solve this system: First, the reexcitation of the STED beam, the probability to excite a molecule from the ground state to the excited state with the STED beam, should be negligible. Second, the system is in temporal equilibrium.

The population of the excited state $N_{1}$, which is proportional to the fluorescence signal, gives information about the efficiency of the depletion process. For the given assumptions the rate equation of the regarded system is

$$
\frac{d N_{1}}{d t}=-\left(k_{f l}+k_{S T E D}\right) N_{1}+k_{e x c} N_{0}
$$

Per definition, the sum over all state populations is normalized, $N_{0}+N_{1}=1$. Because of the temporal equilibrium of the system $\left(\frac{d N_{1}}{d t}=0\right)$ eq. (4.1) results in

$$
0=-\left(k_{f l}+k_{S T E D}\right) N_{1}+k_{e x c}\left(1-N_{1}\right) \Leftrightarrow N_{1}=\frac{k_{e x c}}{k_{f l}+k_{e x c}+k_{S T E D}}
$$

The suppression factor $\eta$ is defined as

$$
\eta=\frac{N_{1}}{\left.N_{1}\right|_{k_{S T E D}=0}}=\frac{1}{1+\frac{k_{S T E D}}{k_{e x c}+k_{f l}}}
$$

The saturation intensity $I_{S}$ is defined as the STED intensity where half of molecules are quenched (same definition as for the pulsed system). With $k_{S T E D}=\sigma_{S T E D} I_{S T E D}, \sigma_{S T E D}$ 
is defined as the cross section for stimulated emission and eq. (4.3) becomes

$$
\eta\left(I_{S}\right)=1 / 2=\frac{k_{e x c}+k_{f l}}{k_{e x c}+k_{f l}+\sigma_{S T E D} I_{S}} \Longleftrightarrow I_{S}=\frac{k_{e x c}+k_{f l}}{\sigma_{S T E D}}
$$

Eq. (4.4) shows one major difference between the $\mathrm{CW}$ mode and the pulsed mode. Due to $\mathrm{CW}$ excitation, the transitions for excitation, fluorescence and stimulated emission can not be separated in time. This is why the saturation intensity depends on the excitation rate $k_{\text {exc }}$.

Assuming a relative excitation saturation of $10 \%$, which is convenient for most image acquisitions, the ratio between $k_{f l}$ and $k_{e x c}$ can be calculated by eq. (4.2):

$$
N_{1}\left(k_{S T E D}=0\right)=\frac{k_{e x c}}{k_{e x c}+k_{f l}}=0.1 \Leftrightarrow k_{e x c}=\frac{1}{9} k_{f l}
$$

This calculation shows that it is justifiable to set $k_{\text {exc }} \ll k_{f l}$, when the excitation saturation is below $10 \%$. Per definition, the probability to emit a photon by relaxing from the excited state to the ground state is the inverse of the lifetime of the excited state, $k_{f l}=1 / \tau_{f l}$, it follows

$$
I_{S}=1 /\left(\tau_{f l} \sigma_{S T E D}\right)
$$

Consider the equation (2.5) from chapter 2.2, which states that the saturation intensity for the pulsed mode is $I_{S}{ }^{\text {pulsed }}=\frac{\ln 2}{\tau \sigma_{S T E D}}$. This is valid for one pulse of a given pulse sequence, but to compare it to the $\mathrm{CW}$ mode it must be converted into the average intensity via $I_{S}{ }^{\text {pulsed }}=$ $\frac{\Gamma}{\tau} \bar{I}_{S}{ }^{\text {pulsed }}$ with the pulse separation $\Gamma$ and the pulse width assuming a rectangle pulse with the width $\tau$. With this the ratio of the saturation intensity in the pulsed mode and the one in the $\mathrm{CW}$ mode (equation (4.6) is:

$$
\frac{{\overline{I_{S}}}^{\text {pulsed }}}{I_{S}{ }^{C W}}=\frac{\ln 2}{\sigma_{S T E D} \Gamma} \cdot \sigma_{S T E D} \tau_{f l}=\frac{\ln 2 \tau_{f l}}{\Gamma}
$$

For the realization of the $\mathrm{CW}$ concept, a factor of $\frac{\Gamma}{\ln 2 \tau_{f l}}$ more average power in the $\mathrm{CW}$ mode is necessary to achieve the same depletion efficiency as in the pulsed mode. Experimental data to validate this relation will follow in the following chapters.

\subsection{Experimental Results}

\subsubsection{Experimental Setup}

The same setup as described in chapter 2.3.1 was used, except for the following modifications of the laser sources: The excitation laser diode was changed to a $\mathrm{CW}$ fiber coupled laser source (FibreTec, AMD technologies, Germany) with a wavelength of $635 \mathrm{~nm}$. For STED the Titanium:Sapphire-laser were set to $\mathrm{CW}$ mode by changing the laser cavity from the main to the auxiliary cavity. 


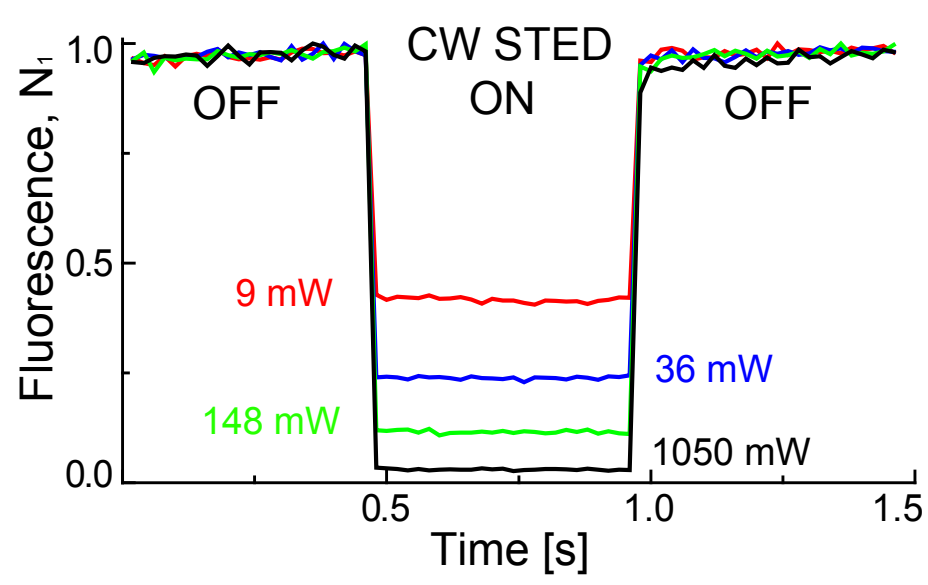

Figure 4.1: Fluorescence signal of Atto $647 \mathrm{~N}$ dye solution with a periodic switching on and off of the CW STED beam.

\subsubsection{First Depletion Measurements}

The first (and probably most important) measurement is to test whether the fluorescence of a specific dye can be depleted by applying a STED beam, because it is the key phenomenon for high resolution measurements [36].

For these measurements the fluorescence signal was collected from a solution of the dye Atto $647 \mathrm{~N}$ (figure 4.1). The time trace over a few seconds is shown with periodic on and off switching of the CW STED laser. A strong reduction of the fluorescence signal can clearly be observed. With increasing power of the CW STED beam, the fluorescence signal is increasingly reduced. This measurement shows the ability to perform high resolution measurements with $\mathrm{CW}$ laser sources.

To validate the relation between the saturation intensity in the pulsed and in the CW STED mode, as postulated in the previous section, quantitative STED depletion measurements were established. For this measurement a sample of fixed fluorescent spheres with a diameter of $24 \mathrm{~nm}$ (already used in previous chapters) was used to measure the STED depletion curve in the $\mathrm{CW}$ as well as in the pulsed mode. For canceling out any kind of geometric effects of the Gaussian beam shapes, the fluorescence depletion was measured in the center of a single isolated bead (for details see appendix A.3). Figure 4.2 shows the resulting curves for these measurements, with the red circles representing the $\mathrm{CW}$ data with the corresponding bottom $\mathrm{x}$-axis and the blue triangles showing the measured data points for the pulsed mode (top x-axis). The shape of the two depletion curves matches each other very well with the two different corresponding x-axes.

A $76 \mathrm{MHz}$ system was used for the pulsed measurement. The dye in the fluorescent beads has a lifetime of $\tau_{f l}=3.77 \mathrm{~ns}$. With these values the power relations between the $\mathrm{CW}$ power and the pulsed average power ends in $I_{S}^{C W}=5.0 \cdot \bar{I}_{S}^{\text {pulsed }}$. For a perfect overlay of the two depletion curves in figure 4.2, a factor of 3.6 was multiplied to the pulsed average power. The mismatch of the two factors might be explained by experimental details. In the pulsed mode, the STED pulse width is about 4 times longer as the excitation pulse. To get 


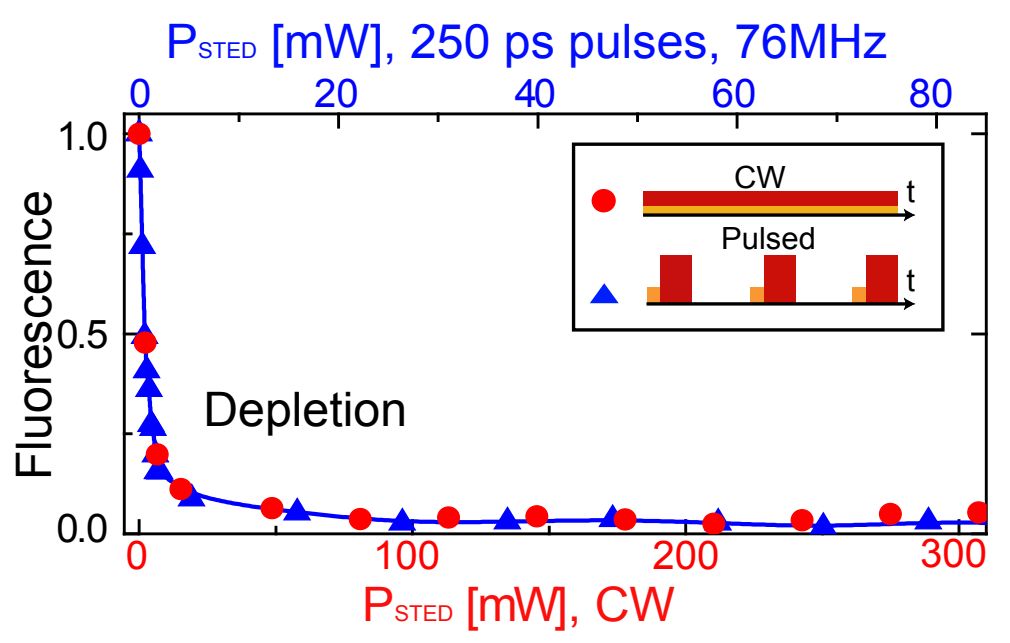

Figure 4.2: STED depletion curves for the CW mode (blue circles, bottom $\mathrm{X}$-axis) and the pulsed mode (blue triangles, top $\mathrm{x}$-axis) measured on the same sample (crimson fluorescent spheres).

the optimal depletion effect, the two pulses overlap in time, meaning that during the STED pulse some molecules are excited by the excitation pulse, which does not support a time separation of these processes. The spectrum of the STED light is also different. Generating short pulses (about $100 \mathrm{fs}$ ) leads to a broad spectrum (about $10 \mathrm{~nm}$ in width), that gets even more elongated by focusing into the single mode fiber, due to non linear effects. The CW spectrum is much narrower (a few $\mathrm{nm}$ ), resulting in different cross sections for stimulated emission. Furthermore, the conversion of the power per pulse to the average power does not take into account the Gaussian pulse shapes. For a correction of this effect, a multiplicative factor of 1.064 has to be incorporated into the pulsed power. The derivation of the saturation intensity in the pulsed mode was based on the assumption of a mono-exponential suppression. Therefore, the experimental estimation of $I_{S}$ might be slightly compromised by higher exponential terms that occur due to orientational effects [64]. However, taking all the mentioned arguments into account, the general relation between the required average powers in the pulsed and in the $\mathrm{CW}$ mode for the same magnitude of the depletion was confirmed by the experimental data.

\subsubsection{High Resolution Measurements with CW STED}

After the validation of the fact that it is possible to deplete excited molecules with $\mathrm{CW}$ STED very efficiently, resolution measurements were performed. For these investigations the $24 \mathrm{~nm}$ fluorescent beads were used again and the absolute value for the resolution was measured. Figure 4.3 presents high resolution measurements on these fluorescent beads. The measurement shows resolution enhancement in the lateral directions using the phase mask $P_{2 D}$ (see definitions in chapter 2.1). The confocal image (a) shows large objects instead of the single beads that are visible in the STED counterpart (b). The profile along the dashed 

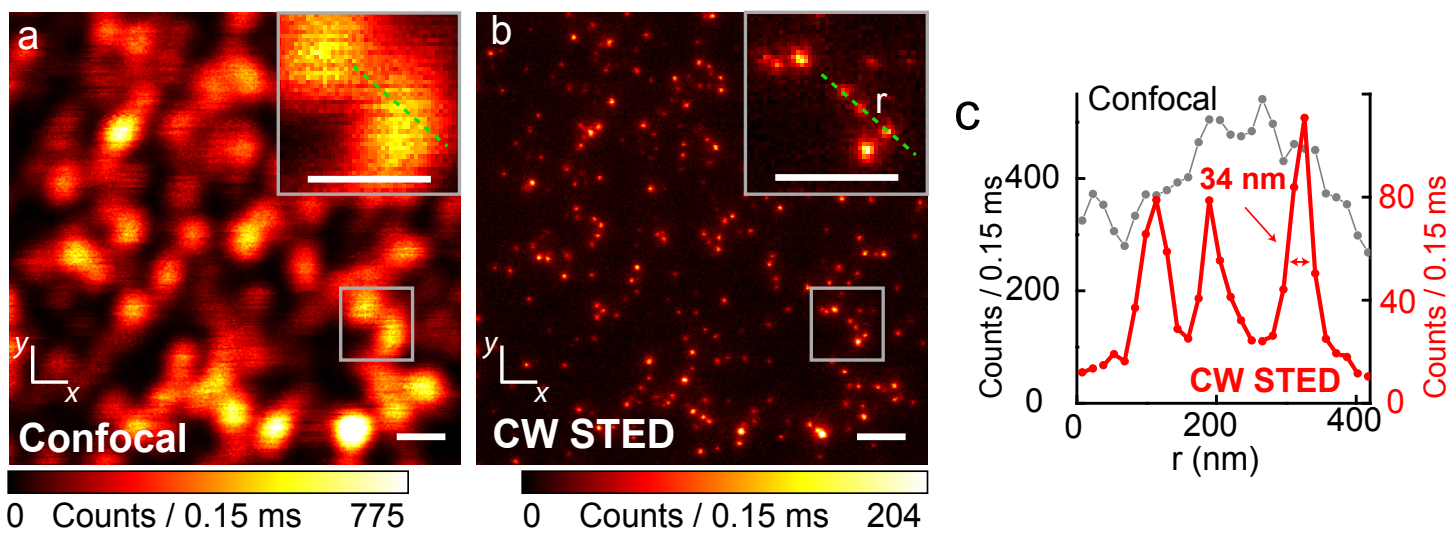

Figure 4.3: High resolution imaging with CW STED. The raw data of the confocal image in the lateral plane (a) shows a cloudy image of the beads while in the STED counterpart (b) nearly every single bead could be identified. A profile (c) along the dashed line in the insets clarifies this fact. Scale bars $500 \mathrm{~nm}$.

line in the insets features three individual beads resolved in the STED profile and no substructure in the profile of the confocal data. The resolution of the microscope was analyzed by measuring the mean of the FWHM of the Gaussian, which was fitted to the intensity profile of several isolated beads. With the given bead size of $24 \mathrm{~nm}$ diameter, the resolution, which is the FWHM of the effective PSF, could be calculated (detailed description in appendix A.2. It was measured to be $29 \mathrm{~nm}$ in the lateral directions, which corresponds to a gain in resolution over the confocal mode of 8 .

Also the achievable resolution along the optical axis was determined by a bead measurement. For these investigations the helical phase mask $P_{2 D}$ was replaced with the axial one, $P_{3 D}$. Two cover slips with $43 \mathrm{~nm}$ fluorescent beads attached were brought close together. In the confocal imaging mode, no structure about the real sample can be explored (figure 4.4 (a)), while in the STED counterpart the presence of two single bead layers is clearly evident (b). Profiles along the dashed lines show a resolution of the CW STED technique in the axial direction of $\approx 160 \mathrm{~nm}$, which is a 4-fold enhancement relative to the confocal mode.

The resolution measurements show the huge ability of CW STED for high resolution microscopy. The required intensities for the best resolutions are still in an achievable regime.

\subsubsection{High Resolution Measurements in Biological Samples}

The capability of CW STED for performing high resolution microscopy has been shown in the previous section. A very important issue regarding the power of an imaging technique is the feasibility to implement this technique for biological samples.

For these measurements, antibodies decorated with the dye Atto $647 \mathrm{~N}$ were used to immunolabel biological tissues. For proving the capability of CW STED microscopy in the lat- 


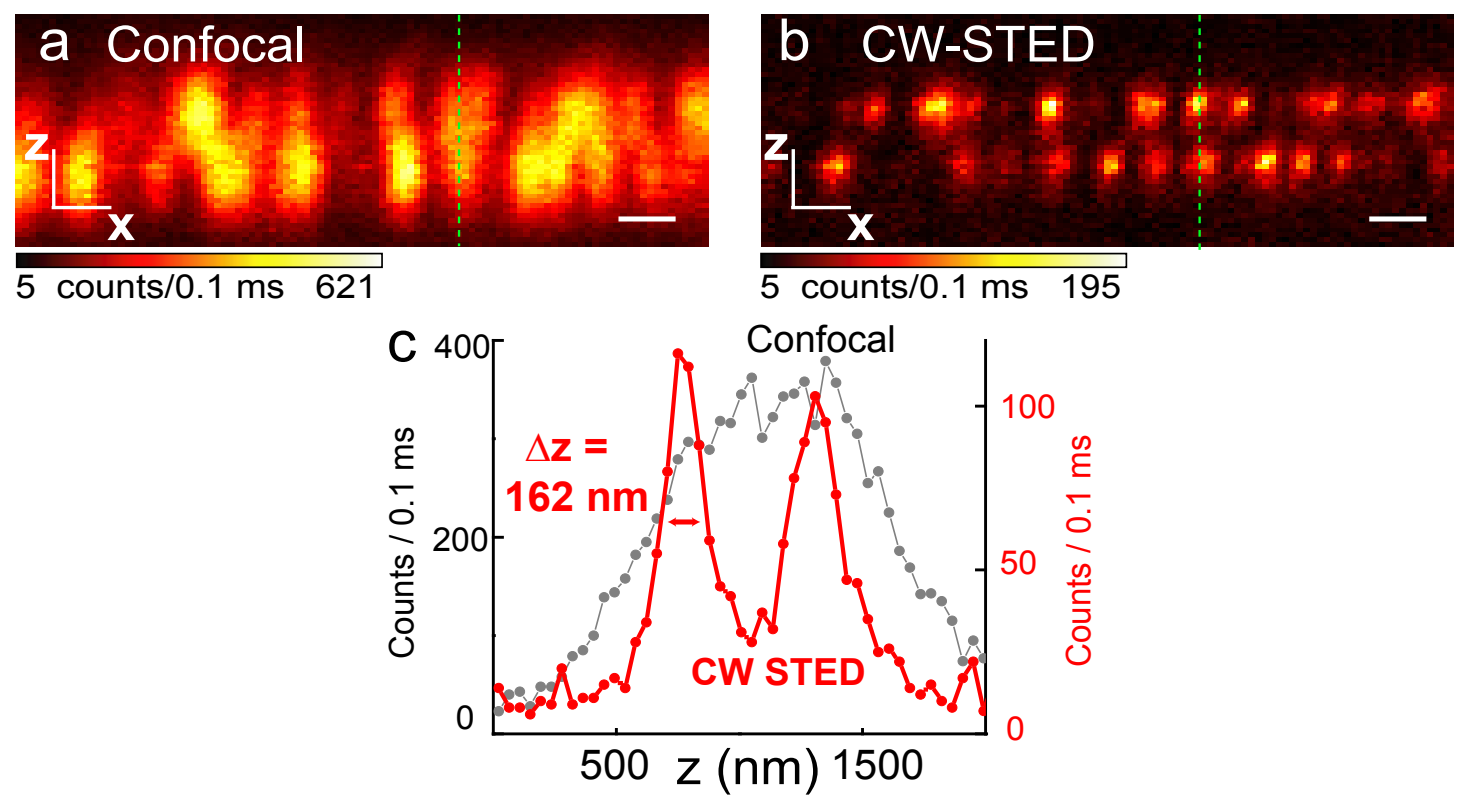

Figure 4.4: 3D super resolution in axial optical sections. Confocal $x z$-section (a) in comparison to the corresponding CW STED image (b) providing the information of two separate layers. The profile along the dashed line (c) emphasis this fact. Scale bars $500 \mathrm{~nm}$

eral directions, micrographs of neurofilaments in human neuroblastoma [65] were stained 1 ] Neurofilaments are intermediate filaments found specifically in neurons and they play an important role in the mechanisms of neuro degenerative diseases like Alzheimers and Parkinsons disease.

Figure 4.5 shows the confocal (a) as well as the high resolution CW STED (b) measurements. Single protein clusters can be resolved in the CW STED mode, whereas in the confocal image any substructure of the sample can not be resolved.

Point like emitters, in this case single stained antibodies, were measured with the same measurement settings to estimate the resolution capability of the microscope. The analysis of this recording established a resolution of $\approx 230 \mathrm{~nm}$ in the confocal mode and $\approx 52 \mathrm{~nm}$ in the CW STED mode, which corresponds to resolution gain factor of $\approx 4.5$ relative to the confocal mode in biological samples.

In the previous section it was shown that an enhanced resolution can be achieved also in the axial direction. In chapter 3 the onset of photobleaching was discussed as a limiting factor in $3 \mathrm{D}$ imaging. The question therefore is whether recording of 3D data stacks in biological tissue with CW STED microscopy can be achieved or if it is limited by bleaching?

For this test, a sample of cell nuclei very similar to that discussed in chapter 3.2.4 was prepared and the proteins Lamin A and C were immunolabeled with the dye Atto 647N. A surface rendered image of the data stack is shown in the center of figure 4.6(b). The insets

\footnotetext{
${ }^{1}$ Samples prepared by R. Medda, Department of Nanobiophotonics, Göttingen
} 


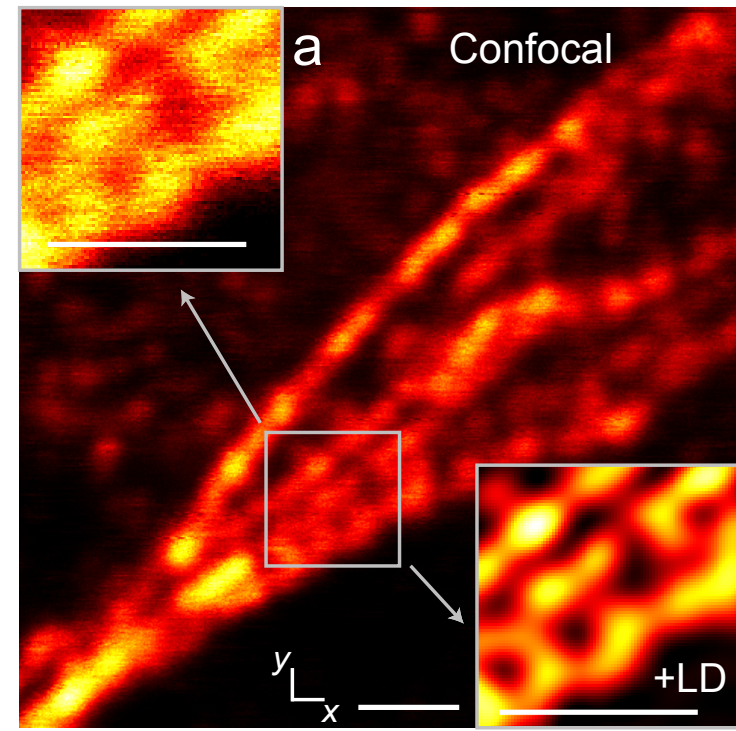

10 counts/0.1 ms 1303

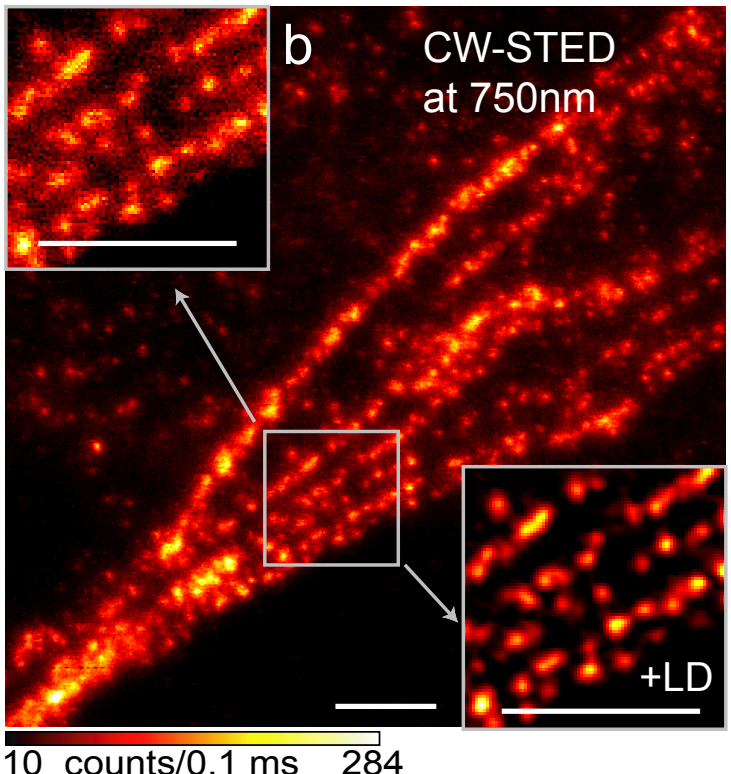

10 counts $/ 0.1 \mathrm{~ms} 284$

Figure 4.5: Immunofluorescence CW STED microscopy of neurofilaments in human neuroblastoma. No substructure can be resolved in the confocal image (a) even with linear deconvolution in the lower right inset. The CW STED image resolves single clusters of proteins (b). Scale bars $1 \mu \mathrm{m}$
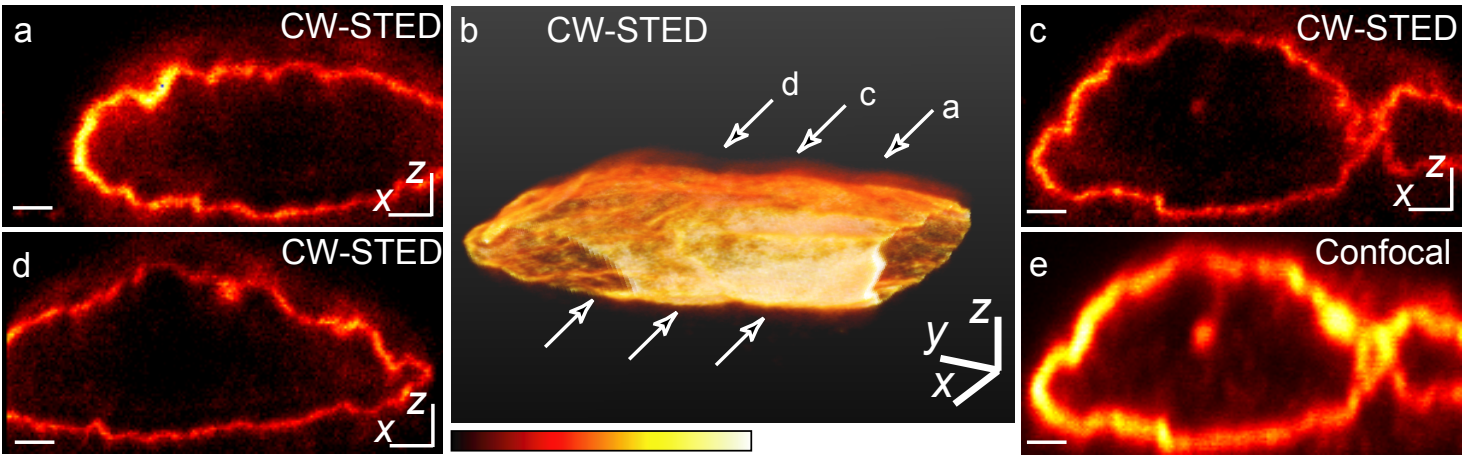

Figure 4.6: 3D high resolution imaging of the nuclear lamina. (b) A surface rendered view of the 3D data stack. (a,c,d) Three $x z$-sections of the raw data stack marked by white arrows in (b). (e) confocal counterpart to (c). Scale bars $1 \mu m$. 
$(\mathrm{a}, \mathrm{c}, \mathrm{d})$ present the raw data of the acquired $x z$-sections along the areas marked by the white arrows in (c). For reference, a confocal $x z$-section (e) is displayed to compare it with the same $x z$-section in the CW STED mode (c). A benefit in resolution can be directly observed. The axial resolution, measured with the FWHM of the nuclear membrane in the $z$-direction, was analyzed to below $200 \mathrm{~nm}$, which corresponds to an enhancement factor of 3 relative to the confocal mode. The bleaching rate during the data acquisition was in a reasonable regime allowing 3D imaging with CW STED microscopy by taking $200 x z-$ sections with a pixel size of $70 \mathrm{~nm}$.

\subsection{Discussion and Summary}

The bleaching channel from the triplet state to higher excited triplet states has previously been identified as the major source for photobleaching for many dyes [66] [67][68]. Nevertheless, the lifetime of the dark triplet state is in the range of $\mu s$, means no difference between exciting the dye molecule with a $76 \mathrm{MHz}$ pulsed laser source (about $13 \mathrm{~ns}$ pulse separation) or with a CW laser. For dyes with a very large triplet yield, typically present in the visible range, a pulsed laser system with inter pulse separation longer than the lifetime of the triplet state is recommended to circumvent the problem of photobleaching. In between two consecutive pulses the triplet state (or in general a dark state) can relax to the ground state, which excludes bleaching over higher excited triplet states. This has been realized for the excitation in one- and two-photon mode via dark state relaxation ([69]). The implementation of this technique in a high resolution microscope has also already been done ([37]), where the triplet state was identified as the dark state. Therefore the behavior of the dyes in terms of photobleaching in the $\mathrm{CW}$ mode should be the same as for the pulsed mode for the $76 \mathrm{MHz}$ system used in the previous chapters.

Using CW light sources also opens up new possibilities for fast scanning. The disadvantage of using a laser system with a low repetition rate is the high data acquisition time, because in between two consecutive pulses the dye is not excited and hence gives no fluorescence signal. For collecting enough fluorescence photons the integration time has to be set to a high value especially for a small effective PSF. One solution is to go again to light sources with a higher repetition rate [70], accepting the higher bleaching rates. Then the fastest solution is the $\mathrm{CW}$ light source as it produces the highest photon flux, because after emitting a photon a dye molecule can be immediately excited again. However, it has to be mentioned, that the calculations showed an important difference to the pulsed mode: the saturation intensity depends on the excitation level. The maximal scanning speed is always limited by the detected fluorescence signal. To gain the scanning speed, the signal has to be enlarged by increasing the excitation power followed by an increase of the saturation intensity. To compensate for this a higher STED power has be applied ending in higher bleaching rate. A compromise has to be performed to achieve the optimal efficiency for scanning speed and resolution, respectively. 
In this chapter the use of $\mathrm{CW}$ laser sources for the excitation and depletion process has been shown. A key phenomenon for sub-diffraction microscopy is the presence of a depletion process. Therefore depletion measurements in a dye solution as well as on fluorescent beads was performed. As a result the measurement showed the ability of high resolution measurements using $\mathrm{CW}$ laser sources. The required average powers were in the same range as theoretically expected. The reasons for the mismatch were discussed. The resolution measurements on technical bead samples showed a resolution of $29 \mathrm{~nm}$ in the lateral and $170 \mathrm{~nm}$ in the axial direction. The benefit in biological samples has been pointed out by recording the neurofilaments in human blastoma. The corresponding resolution measurements yielded $51 \mathrm{~nm}$ in the lateral and below $200 \mathrm{~nm}$ in the axial direction. The critical parameter of photobleaching has not turned out to get in the way of 3D imaging. With the recording of a nuclear laminar it has been shown that this microscopy method has a significant potential for 3D imaging. The fact of not having excessive photobleaching has also been validated for other dyes in this publication [71]. In addition, measurements on biological samples with the visible dye ATTO 565 are shown in the appendix A.4.2.

In conclusion, the use of $\mathrm{CW}$ laser sources for high resolution microscopy has been proved for several dyes. The resulting simplicity of the setup gives in principle every scanning microscope the possibility of implementing sub-diffraction resolution capabilities. 


\section{Conclusion and Outlook}

This thesis shows a way to achieve resolution enhancement in fluorescence microscopy breaking the diffraction barrier in all spatial directions. Investigations on biological and artificially structured samples have been presented which demonstrate new possibilities for efficient structural analysis in three dimensions. In addition, the introduction of CW STED microscopy cleared the way to implement STED microscopy in nearly all existing imaging systems.

The implementation of a novel phase plate that induces a helical phase retardation to the depletion beam improved the quality of the depletion pattern in terms of the residual intensity in the center of the focal spot. With an experimentally acquired value for the residual intensity of about $0.5 \%$ relative to maximum values in the rim of the doughnut, high resolution measurements can be performed without any restrictions caused by a non-perfect zero, as mentioned in previous work [42]. With the high quality of the depletion pattern, the theoretically predicted modified Abbe resolution formula of the microscope has been experimentally confirmed. Thereby, a resolution below $25 \mathrm{~nm}$ in the lateral directions was achieved with the helical phase plate applied to the depletion beam. Applying the axial phase mask to the STED beam yielded a resolution of $94 \mathrm{~nm}$.

In these experiments the resolution was limited by the available STED laser power. Further progress is expected from recent developments in laser technology and dye chemistry. Dyes featuring a long Stokes shift can be depleted more efficiently since the STED wavelength can be shifted closer to the emission maximum without a significant reexcitation probability. The resulting higher saturation factor yields a further enhanced resolution. The development of color-tunable dyes with large cross sections and high quantum yields [72] is in progress and can be combined with a supercontinuum laser for multiple color imaging.

As the microscope's resolution is getting close to the size of the marker molecules, labeling techniques have to be reconsidered. Immunolabeling is one of the standard labeling technique for biological imaging. As an illustration consider the complex of a primary antibody coupled to 2-4 secondary antibodies stained with 3-7 dye molecules which results in a total size in the range of $15 \mathrm{~nm}$ [39] providing artifacts during a high resolution imaging process. An alternative way of labeling proteins is genetically adding an amino acid motif like tetracysteine-tag which binds the marker molecules FLAsH [73] or ReAsH [74]. Due to the small marker size of below $1 \mathrm{~nm}$ live cell imaging is feasible with this labeling technique[75].

For 3D imaging the effective focal volume has been effectively reduced by a factor of 125 relative to the confocal focal volume by incoherently combining two different depletion 
patterns. The improved resolution achieved with these settings was measured to be $43 \mathrm{~nm}$ in the lateral directions and $125 \mathrm{~nm}$ in the axial direction in a 3D data stack. The benefit of the reduced focal volume has been used for the imaging of self organized colloidal crystals with a particle size of $200 \mathrm{~nm}$. Since the confocal resolution especially in the axial direction is not sufficient, structures built up by particles of this size have not been accessible yet. Imaging up to 7 layer crystals and identifying the structure via a localization method, where no prior knowledge is necessary, have been performed. In biological samples the nuclear lamina within a three dimensional data stack with an enhanced resolution in all directions was imaged.

The three dimensional resolution enhancement demonstrated in this thesis clearly show the ability to perform the structural analysis of technical and biological samples with STED microscopy. However, unlike in the previous power limited measurements, the resolution is now limited by the onset of photobleaching. The further development of this technique is dependent on the mitigation of this phenomenon. Work in this area would thus focus on optimization efforts such as, once again, the improvement of the dye itself or by the addition of antifading reagents like triplet quenchers. These quenchers reduce the incidence of bleaching by depleting the triplet state via energy transfer thus preventing bleaching associated with excited triplet states. Despite the challenges of photobleaching, the three dimensional crystal imaging of densely packed colloidal crystals has been demonstrated for particle sizes of $200 \mathrm{~nm}$ in diameter. The ability to image structures on the nanometer scale has strong implications for the colloidal sciences since the relative magnitudes of the attractive and repulsive forces that govern the process of self-assembly are size dependent. In particular, effects specific to the nanoscale regime have been observed, where the width of the screening layer is commensurate with the size of the colloidal particles, such as the formation of nonclose-packed diamond-like lattices [76]. Imaging crystal structures consisting of two or more different particles sizes or colors gives new information about model systems. Investigating dynamic effects within a fast scanning microscope can offer new information about self-assembly or crystal melting.

The use of CW laser sources for excitation and depletion has been identified as a convenient and inexpensive alternative for high resolution imaging. With the simplified setup a lateral resolution of below $30 \mathrm{~nm}$ in artificial and about $50 \mathrm{~nm}$ in biological samples was achieved. An axial resolution of below $170 \mathrm{~nm}$ in $x z$-sections of artificially structured samples and about $200 \mathrm{~nm}$ in three dimensional imaging of biological tissue has been shown.

Implementing $\mathrm{CW}$ lasers results in an experimental setup that does not require any pulse optimization optics or additional electrical equipment. The lasers themselves are much cheaper, especially for the excitation laser as a conventional laser diode - like a laser pointer - satisfies all requirements. The major benefit of CW STED microscopy can be achieved in combination with a fast scanning setup which is commonly used in standard confocal microscopy. A fast scanning system with a pulsed laser system has already been realized [70], but the enhanced photon flux due to the absence of inter pulse breaks in the $\mathrm{CW}$ mode can further improve fast scanning high resolution techniques. However, the scanning speed of high resolution microscopes is limited by the detected signal. To boost the scanning speed, 
the excitation rate has to be increased. But the higher excitation rate has to be compensated by an increased depletion rate in form of a higher STED intensity causing enhanced bleaching. A compromise between these parameters has be found to achieve optimal imaging performance.

The combination of the fast scanning ability and the use of low peak intensities in the depletion beam also should render live cell imaging feasible. Because of the availability of numerous CW laser sources at various wavelengths multi-color imaging (as it has already been shown for pulsed systems [77]) should be possible in the near future.

The ultimate goal of having a multi-color optical microscope with nanoscale resolution combined with fast scanning device, should be achieved in the near future. 


\section{Bibliography}

[1] G. Binnig, C.F. Quate, and Ch. Gerber. Atomic force microscope. Phys. Rev. Lett., 56:930-933, 1986.

[2] G. Binnig and H. Rohrer. Scanning tunneling microscopy - from birth to adolescence. Rev. Mod. Phys., 59(3):615-625, 1987.

[3] D. W. Pohl and D. Courjon. Near field optics. Kluwer, Dordrecht, 1993.

[4] E. Abbe. Beiträge zur Theorie des Mikroskops und der mikroskopischen Wahrnehmung. Arch. Mikr. Anat., 9:413-420, 1873.

[5] T. Wilson (ed.). Confocal microscopy. Academic Press Ltd., London, 1990.

[6] A. Miyawaki, J. Llopis, R. Heim, J.M. McCaffery, and R.Y. Tsien. Fluorescent indicators for ca2+ based on green fluorescent proteins and calmodulin. Nature, 388:882-887, 1997.

[7] A. Miyawaki, A. Sawano, and T. Kogure. Lighting up cells: labelling proteins with fluorophores. Nature Cell Biology, 5:S1-S7, 2003.

[8] S. M. Mansfield and G. S. Kino. Solid immersion microscope. Applied physics letters, $57: 2615,1990$.

[9] Q. Wu, G. D. Feke, R. D. Grober, and L. P. Ghislain. Realization of numerical aperture 2.0 using a gallium phosphide solid immersion lens. Applied physics letters, 75:4064, 1999.

[10] S. W. Hell and E. H. K. Stelzer. Fundamental improvement of resolution with a 4pi-confocal fluorescence microscope using two-photon excitation. Opt. Commun., 93:277-282, 1992.

[11] H. Gugel, J. Bewersdorf, S. Jakobs, J. Engelhardt, R. Storz, and S.W. Hell. Cooperative 4pi excitation and detection yields 7-fold sharper optical sections in live cell microscopy. Biophys. J., 87:4146-4152, 2004.

[12] A. Egner, S. Verrier, A. Goroshkov, H.-D. Söling, and S.W. Hell. 4pi-microscopy of the golgi apparatus in live mammalian cells. J. Struct. Biol., 147(1):70-76, 2004.

[13] A. Egner and S. W. Hell. Fluorescence microscopy with super-resolved optical sections. Trends in Cell Biology, 15(4):207-215, 2005. 
[14] M. Lang, T. Müller, J. Engelhardt, and S. W. Hell. 4pi microscopy of type a with 1-photon excitation in biological fluorescence imaging. Optics Express, 15(5):24592467, 2007.

[15] K. Konig, Y. Tadir, P. Patrizio, M. W. Berns, and B. J. Tromberg. Effects of ultraviolet exposure and near infrared laser tweezers on human spermatozoa. Human Reproduction, 11(10):2162-2164, 1996.

[16] L. Reimer. Scanning electron microscopy. Springer Series in Optical Sciences. Springer-Verlag, Berlin, Heidelberg, New York, Tokyo, 1985.

[17] W. Baumeister. Electron tomography: towards visualizing the molecular organization of the cytoplasm. Curr. Opin. Struct. Biol., 12:679-684, 2002.

[18] M. Minsky. Microscopy apparatus, US patent 3,013,467, 1961.

[19] T. Wilson and C. J. R. Sheppard. Theory and practice of scanning optical microscopy. Academic Press, New York, 1984.

[20] T. Wilson and A. R. Carlini. The effect of aberrations on the axial response of confocal imaging systems. J. Microsc., 154(3):243-256, 1989.

[21] A. Egner, V. Andresen, and S.W. Hell. Comparison of the axial resolution of practical nipkow-disk confocal fluorescence microscopy with that of multifocal multiphoton microscopy: theory and experiment. J. Microsc., 206:24-32, 2002.

[22] W. Denk, J. H. Strickler, and W. W. Webb. Two-photon laser scanning fluorescence microscopy. Science, 248:73-76, 1990.

[23] E. Betzig, G.H. Patterson, R. Sougrat, O.W. Lindwasser, S. Olenych, J.S. Bonifacino, M.W. Davidson, J. Lippincott-Schwartz, and H.F. Hess. Imaging intracellular fluorescent proteins at nanometer resolution. Science, 313(5793):1642-1645, 2006.

[24] A. Egner, C. Geisler, C. von Middendorff, H. Bock, D. Wenzel, R. Medda, M. Andresen, A. Stiel, S. Jakobs, C. Eggeling, A. Schönle, and S. W. Hell. Fluorescence nanoscopy in whole cells by asnychronous localization of photoswitching emitters. Biophys. J., 93:3285-3290, 2007.

[25] M. J. Rust, M. Bates, and X. Zhuang. Sub-diffraction-limit imaging by stochastic optical reconstruction microscopy (STORM). Nature Methods, 3:793-796, 2006.

[26] J. Fölling, V. Belov, R. Kunetsky, R. Medda, A. Schönle, A. Egner, C. Eggeling, M. Bossi, and S. W. Hell. Photochromic rhodamines provide nanoscopy with optical sectioning. Angew. Chem. Int. Ed., 46:6266-6270, 2007.

[27] B. Huang, W. Wang, M. Bates, and X. Zhuang. Three-dimensional super-resolution imaging by stochastic optical reconstruction microscopy. Science Express, 319:810 813, 2008. 
[28] S.W. Hell. Toward fluorescence nanoscopy. Nature Biotechnol., 21(11):1347-1355, 2003.

[29] M. Hofmann, C. Eggeling, S. Jakobs, and S.W. Hell. Breaking the diffraction barrier in fluorescence microscopy at low light intensities by using reversibly photoswitchable proteins. Proc. Natl. Acad. Sci. USA, 102(49):17565-17569, 2005.

[30] M. Andresen, A. C. Stiel, S. Trowitzsch, G. Weber, C. Eggeling, M. C. Wahl, S. W. Hell, and S. Jakobs. Structural basis for reversible photoswitching in dronpa. Proc. Natl. Acad. Sci. USA, 104:13005-13009, 2007.

[31] M. Schwentker, H. Bock, M. Hofmann, S. Jakobs, J. Bewersdorf, C. Eggeling, and S. W. Hell. Wide-field subdiffraction resolft microscopy using fluorescent protein photoswitching. Micr. Res. Tech., 70(3):269-280, 2007.

[32] S. W. Hell and M. Kroug. Ground-state depletion fluorescence microscopy, a concept for breaking the diffraction resolution limit. Appl. Phys. B, 60:495-497, 1995.

[33] S. Bretschneider, C. Eggeling, and S. W. Hell. Breaking the diffraction barrier in fluorescence microscopy by optical shelving. Phys. Rev. Lett., 98:218103, 2007.

[34] S. W. Hell and J. Wichmann. Breaking the diffraction resolution limit by stimulated emission. Opt. Lett., 19(11):780-782, 1994.

[35] A. Einstein. Zur Quantentheorie der Strahlung. Physik. Zeitschr., 18:121-128, 1917.

[36] T.A. Klar and S.W. Hell. Subdiffraction resolution in far-field fluorescence microscopy. Opt. Lett., 24(14):954-956, 1999.

[37] G. Donnert, J. Keller, R. Medda, M. A. Andrei, S. O. Rizzoli, R. Lührmann, R. Jahn, C. Eggeling, and S. W. Hell. Macromolecular-scale resolution in biological fluorescence microscopy. Proc. Natl. Acad. Sci. USA, 103(31):11440-11445, 2006.

[38] M. Dyba and S.W. Hell. Focal spots of size 1/23 open up far-field fluorescence microscopy at $33 \mathrm{~nm}$ axial resolution. Phys. Rev. Lett., 88:163901, 2002.

[39] M. Dyba, S. Jakobs, and S.W. Hell. Immunofluorescence stimulated emission depletion microscopy. Nature Biotechnol., 21(11):1303 - 1304, 2003.

[40] T.A. Klar, E. Engel, and S.W. Hell. Breaking Abbe's diffraction resolution limit in fluorescence microscopy with stimulated emission depletion beams of various shapes. Phys. Rev. E, 64:066613, 1-9, 2001.

[41] T. A. Klar. Progress in Stimulated Emission Depletion Microscopy. PhD thesis, Rupertus Carola University of Heidelberg, 2001.

[42] K. I. Willig. STED microscopy in the visible range. PhD thesis, Rupertus Carola University of Heidelberg, 2006. 
[43] K. I. Willig, S. O. Rizzoli, V. Westphal, R. Jahn, and S. W. Hell. STED-microscopy reveals that synaptotagmin remains clustered after synaptic vesicle exocytosis. Nature, 440(7086):935 - 939, 2006.

[44] R. Kellner, J. Baier, K. I. Willig, S. W. Hell, and F. J. Barrantes. Nanoscale organization of nicotinic acetylcholine receptors revealed by STED microscopy. Neuroscience, 144(1):135-143, 2007.

[45] T. A. Klar, S. Jakobs, M. Dyba, A. Egner, and S. W. Hell. Fluorescence microscopy with diffraction resolution limit broken by stimulated emission. Proc. Natl. Acad. Sci. USA, 97:8206-8210, 2000.

[46] L. Kastrup, H. Blom, C. Eggeling, and S. W. Hell. Fluorescence fluctuation spectroscopy in subdiffraction focal volumes. Phys. Rev. Lett., 94:178104, 2005.

[47] B. Richards and E. Wolf. Electromagnetic diffraction in optical systems ii. structure of the image field in an aplanatic system. Proc. R. Soc. Lond. A, 253:358-379, 1959.

[48] V. Westphal and S.W. Hell. Nanoscale resolution in the focal plane of an optical microscope. Phys. Rev. Lett., 94:143903, 2005.

[49] P. Dedecker, B. Muls, J. Hofkens, J. Enderlein, and J. I. Hotta. Orientational effects in the excitation and de-excitation of single molecules interacting with donut-mode laser beams. Optics Express, 15:3372-3383, 2007.

[50] J. J. Sieber, K. I. Willig, C. Kutzner, C. Gerding-Reimers, B. Harke, G. Donnert, B. Rammner, C. Eggeling, S. W. Hell, H. Grubmüller, and T. Lang. Anatomy and dynamics of a supramolecular membrane protein cluster. Science, 317:1072 - 1076, 2007.

[51] J. J. Sieber, K. I. Willig, R. Heintzmann, S. W. Hell, and T. Lang. The snare-motif is essential for the formation of syntaxin clusters in the plasma membrane. Biophys. J., 90:2843-2851, 2006.

[52] J. Keller. Optimal de-excitation patterns for RESOLFT-Microscopy. $\mathrm{PhD}$ thesis, $\mathrm{Ru}-$ pertus Carola University of Heidelberg, 2006.

[53] U. Gasser, E. R. Weeks, A. Schofield, P. N. Pusey, and D. A. Weitz. Real-space imaging of nucleation and growth in colloidal crystallization. Science, 292:258-262, 2001.

[54] A Yethiraj and A van Blaaderen. A colloidal model system with an interaction tunable from hard sphere to soft and dipolar. Nature, 421:513, 2003.

[55] A. Van blaaderen and P. Wiltzius. Real-space structure of colloidal hard-sphere glasses. Science, 270(5239):1177-1179, 1995. 
[56] Y. A. Vlasov, X. Bo, J. C. Sturm, and D. J. Norris. On-chip natural assembly of silicon photonic bandgap crystals. Nature, 414:289-293, 2002.

[57] N. D. Denkov, O. D. Velev, P. A. Kralchevsky, I. B. Ivanov, H. Yoshimura, and K. Nagayama. Mechanism of formation of 2-dimensional crystals from latex-particles on substrates. Langmuir, 8(12):3183-3190, 1992.

[58] N. D. Denkov, O. D. Velev, P. A. Kralchevsky, I. B. Ivanov, H. Yoshimura, and K. Nagayama. 2-dimensional crystallization. Nature, 361(6407):26-26, 1993.

[59] V. Prasad, D. Semwogerere, and E. R. Weeks. Confocal microscopy of colloids. Journal of Physics-Condensed Matter, 19(11), 2007.

[60] A. S. Dimitrov and K. Nagayama. Continuous convective assembling of fine particles into two-dimensional arrays on solid surfaces. Langmuir, 12(5):1303-1311, 1996.

[61] P. Jiang, J. F. Bertone, K. S. Hwang, and V. L. Colvin. Single-crystal colloidal multilayers of controlled thickness. Chemistry of Materials, 11(8):2132-2140, 1999.

[62] L. Gerace and G. Blobel. The nuclear envelope lamina is reversibly depolymerized during mitosis. Cell, 19:277-287, 1980.

[63] A. Hopt and E. Neher. Highly nonlinear photodamage in two-photon fluorescence microscopy. Biophys. J., 80:2029-2036, 2001.

[64] M. Dyba, J. Keller, and S. W. Hell. Phase filter enhanced sted-4pi fluorescence microscopy: theory and experiment. New Journal of Physics, 7, 2005.

[65] A. Yuan, R. A. Nixon, and M. V. Rao. Deleting the phosphorylated tail domain of the neurofilament heavy subunit does not alter neurofilament transport rate in vivo. Neuroscience Letters, 393(2-3):264-268, 2006.

[66] C. Eggeling, J. Widengren, R. Rigler, and C. A. M. Seidel. Photobleaching of fluorescent dyes under conditions used for single-molecule detection: Evidence of two-step photolysis. Anal. Chem., 70:2651-2659, 1998.

[67] J. Widengren, Ü. Mets, and R. Rigler. Fluorescence correlation spectroscopy of triplet states in solution: A theoretical and experimental study. J. Phys. Chem., 99:1336813379, 1995.

[68] C. Eggeling, A. Volkmer, and C. A. M. Seidel. Molecular photobleaching kinetics of rhodamine $6 \mathrm{~g}$ by one- and two-photon induced confocal fluorescence microscopy. ChemPhysChem, 6:791-804, 2005.

[69] G. Donnert, C. Eggeling, and S. W. Hell. Major signal increase in flurorescence microscopy through dark-state relaxation. Nat. Methods, 4(1):81-86, 2007. 
[70] V. Westphal, M A Lauterbach, A Di Nicola, and S. W. Hell. Dynamic far-field fluorescence nanoscopy. New J. Phys., 9:435, 2007.

[71] K. I. Willig, B. Harke, R. Medda, and S. W. Hell. STED microscopy with continuous wave beams. Nature Methods, 4(11):915-918, 2007.

[72] K. Umezawa, Y. Nakamura, H. Makino, D. Citterio, and K. Suzuki. Bright, colortunable fluorescent dyes in the visible-near-infrared region. J. Am. Chem. Soc., 130:1550, 2008.

[73] B.A. Griffin, S.R. Adams, and R.Y. Tsien. Specific covalent labeling of recombinant protein molecules inside live cells. Science, 281:269-272, 1998.

[74] G. Gaietta, T.J. Deerinck, S.R. Adams, J. Bouwer, O. Tour, D.W. Laird, G.E. Sosinsky, R.Y. Tsien, and M.H. Ellisman. Multicolor and electron microscopic imaging of connexin trafficking. Science, 269:503-507, 2002.

[75] M. Andresen, R. Schmitz-Salue, and S. Jakobs. Short tetracysteine tags to beta-tubulin demonstrate the significance of small labels for live cell imaging. Mol. Biol. Cell, 15:5616-5622, 2004.

[76] A. M. Kalsin, M. Fialkowski, M. Paszewski, S. K. Smoukov, K. J. M. Bishop, and B. A. Grzybowski. Electrostatic self-assembly of binary nanoparticle crystals with a diamond-like lattice. Science, 312(5772):420-424, 2006.

[77] G. Donnert, J. Keller, C. A. Wurm, S. O. Rizzoli, V. Westphal, A. Schönle, R. Jahn, S. Jakobs, C. Eggeling, and S. W. Hell. Two-color far-field fluorescence nanoscopy. Biophys. J., 92(8):L67-69L, 2007.

[78] M. Encinas, M. Iglesias, Y. Liu, H. Wang, A. Muhaisen, V. Cena, C. Gallego, and J. X. Comella. Sequential treatment of sh-sy5y cells with retinoic acid and brain-derived neurotrophic factor gives rise to fully differentiated, neurotrophic factor-dependent, human neuron-like cells. Journal of Neurochemistry, 75(3):991-1003, 2000. 


\section{A Appendix}

\section{A.1 Measurement Parameters}

The following table shows the parameters of the presented measurements. The dwell time per pixel was constant at $100 \mu \mathrm{s}$. The specified powers for the excitation beam and the STED beam are including the estimated objective transmission of $66 \%$. Excitation wavelength was set to $635 \mathrm{~nm}$ in all measurements.

\begin{tabular}{c|c|c|c|c|c} 
figure & scan axes & pixel size $/ n m$ & $P_{E x c} / \mu W$ & $P_{S T E D} / \mathrm{mW}$ & $\lambda_{S T E D} / n m$ \\
\hline 2.7 & $\mathrm{x} / \mathrm{y}$ & $15 / 15$ & 5.4 & $240 ; 44 ; 22 ; 6$ & 730 \\
\hline$\overline{2.8}$ & $\mathrm{x} / \mathrm{y}$ & $10 / 10$ & 5.4 & $248 ; 172 ; 107 ; 54 ; 32 ; 22 ; 11 ; 6$ & 730 \\
\hline$\overline{2.9}$ & $\mathrm{x} / \mathrm{z}$ & $20 / 30$ & 5.4 & $274 ; 162 ; 101 ; 51 ; 20 ; 10 ; 5$ & 730 \\
\hline$\overline{3.2}$ & $\mathrm{x} / \mathrm{z} / \mathrm{y}$ & $25 / 50 / 25$ & 3.0 & 82 & 730 \\
\hline$\overline{3.6}$ & $\mathrm{x} / \mathrm{y}$ & $15 / 15$ & 1.5 & 145 & 750 \\
\hline$\overline{3.7}$ & $\mathrm{x} / \mathrm{y} / \mathrm{z}$ & $20 / 20 / 50$ & 2.3 & 44 & 730 \\
\hline 3.10 & $\mathrm{x} / \mathrm{z} / \mathrm{y}$ & $60 / 50 / 60$ & 5.7 & 101 & 750 \\
\hline$\overline{4.3}$ & $\mathrm{x} / \mathrm{y}$ & $15 / 15$ & 11 & 825 & 730 \\
\hline$\overline{4.4}$ & $\mathrm{x} / \mathrm{z}$ & $60 / 43$ & 6 & 752 & 750 \\
\hline$\overline{4.5}$ & $\mathrm{x} / \mathrm{y}$ & $15 / 15$ & 7 & 423 & 750 \\
\hline \hline 4.6 & $\mathrm{x} / \mathrm{z} / \mathrm{y}$ & $60 / 70 / 60$ & 3.8 & 397 & 750
\end{tabular}

\section{A.2 Measuring the Size of the Effective PSF}

For measuring the resolution of a microscope system, samples with fluorescent beads were recorded. The average FWHM of the measured beads were established by fitting a Gaussian to the intensity profile of several isolated beads. If the resolution of the microscope comes close to the original bead size, the resulting FWHM of the bead images is larger than the PSF width. The image is the result of a convolution of the effective PSF with the object (in this case a sphere with a given diameter). The relation between the FWHM of the measured bead size $w$ and the resolution of the microscope $v$ is shown in the bottom graph of figure A.1. Both axes are normalized to the bead diameter $o$. The graph shows that for a FWHM of the image which is 3 times larger than the bead size, no significant difference between the FWHM of the measured beads and the resolution can be expected. For smaller values, the shape of the graph can be fitted to a polynomial. With this polynomial the resolution of the 


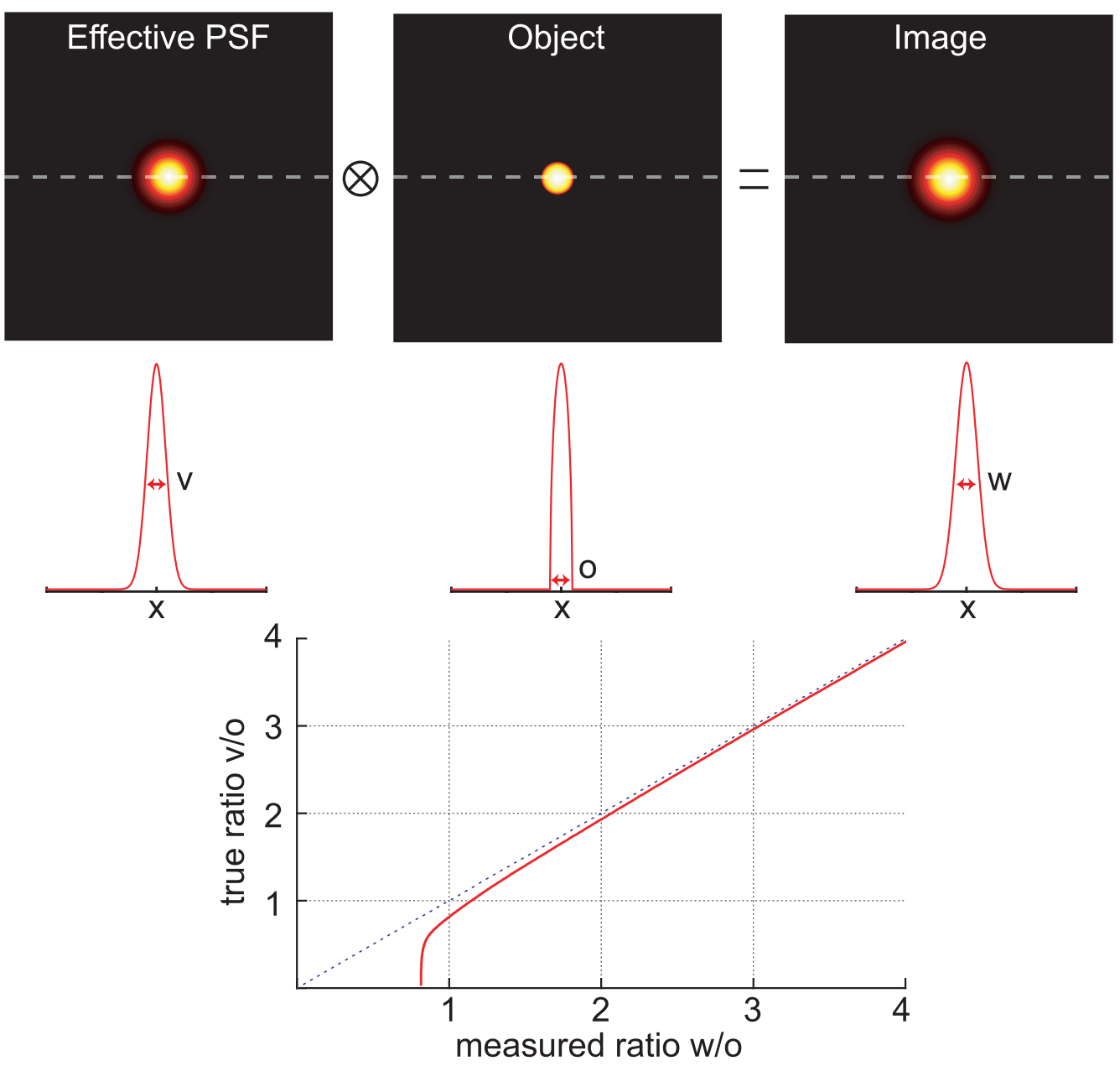

Figure A.1: The image PSF $w$ is the result of the convolution of the effective PSF $v$ with the object $o$. The bottom graph shows the relation between the measured PSF and the effective PSF normalized to the size of the object.

microscope were calculated from the FWHM of the beads:

$$
v / o \cong-0.184(w / o-0.816)^{-0.167}+1.064 w / o, w / o \leq 4
$$

\section{A.3 STED Depletion Measurements}

For performing quantitative STED depletion measurements on fluorescent beads, a diluted sample of these beads was prepared. The measurement modus is described in the following cycles: a confocal image of a $2 \mu \mathrm{m}$ times $2 \mu \mathrm{m}$ area gave the reference. The scan of the same area with both, the excitation and STED beams (no phase mask added), gave the reduction of the fluorescence. A check whether the reexcitation by the STED beam has a significant 
influence was realized by scanning the area with the STED beam only. For every given STED power this cycles was repeated. The resulting STED depletion was calculated by the fraction of the fluorescence signal of the second scan to that of the first scan. To exclude any geometric effects of the structured sample, only the center region of isolated beads was taken into account.

\section{A.4 Additional Measurements}

\section{A.4.1 Microtubuli}

An incoherent combination of patterns produced by two phase masks of the type $P_{1 D}$ leads to an effective resolution enhancement in two directions, when these two phase plates are rotated by $90^{\circ}$. Figure A.2 shows the intermediate filaments microtubuli immunolabeled with the antibody conjugated dye Atto 647N.

\section{A.4.2 CW STED Microscopy}

Figure A.3 shows resolution enhancement in biological samples immunolabeled with the antibody conjugated dye Atto 565 (Atto-Tec, Siegen, Germany). Syntaxin clusters on a membrane sheet of a fixed mammalian cell [50] can be resolved in the CW STED image, while in the confocal measurement no substructure can be assessed.

An image sequence of the previously described sample is shown in figure A.4. After imaging the protein assembly in the confocal mode (a), 5 CW STED images were recorded sequentially (b-f). The total number of counts is indicated beneath the respective image. Another confocal image (g) recorded after the CW STED image series shows a remaining fluorescence of about $50 \%$ compared to (a) clarifying the fact of not having excessive bleaching during CW STED high resolution measurements ${ }^{1}$

\section{A.5 Sample Preparation}

Preparation of the Bead Samples The measured bead samples were prepared by adding a drop of diluted bead solution to a Poly-L-Lysine coated cover slip. After waiting a few minutes, the cover slip was washed with de-ionized water and embedded with Mowiol on the glass slide.

Mowiol 6 g Glycerol AR (\#4094, Merck, Darmstadt, Germany), 2.4 g Mowiol 4-88

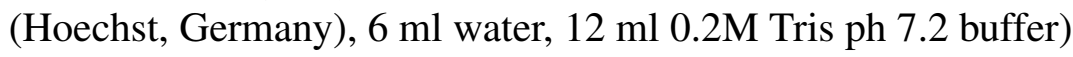

\footnotetext{
${ }^{1}$ Measurements done by K. I. Willig, Department of Nanobiophotonics, Göttingen
} 


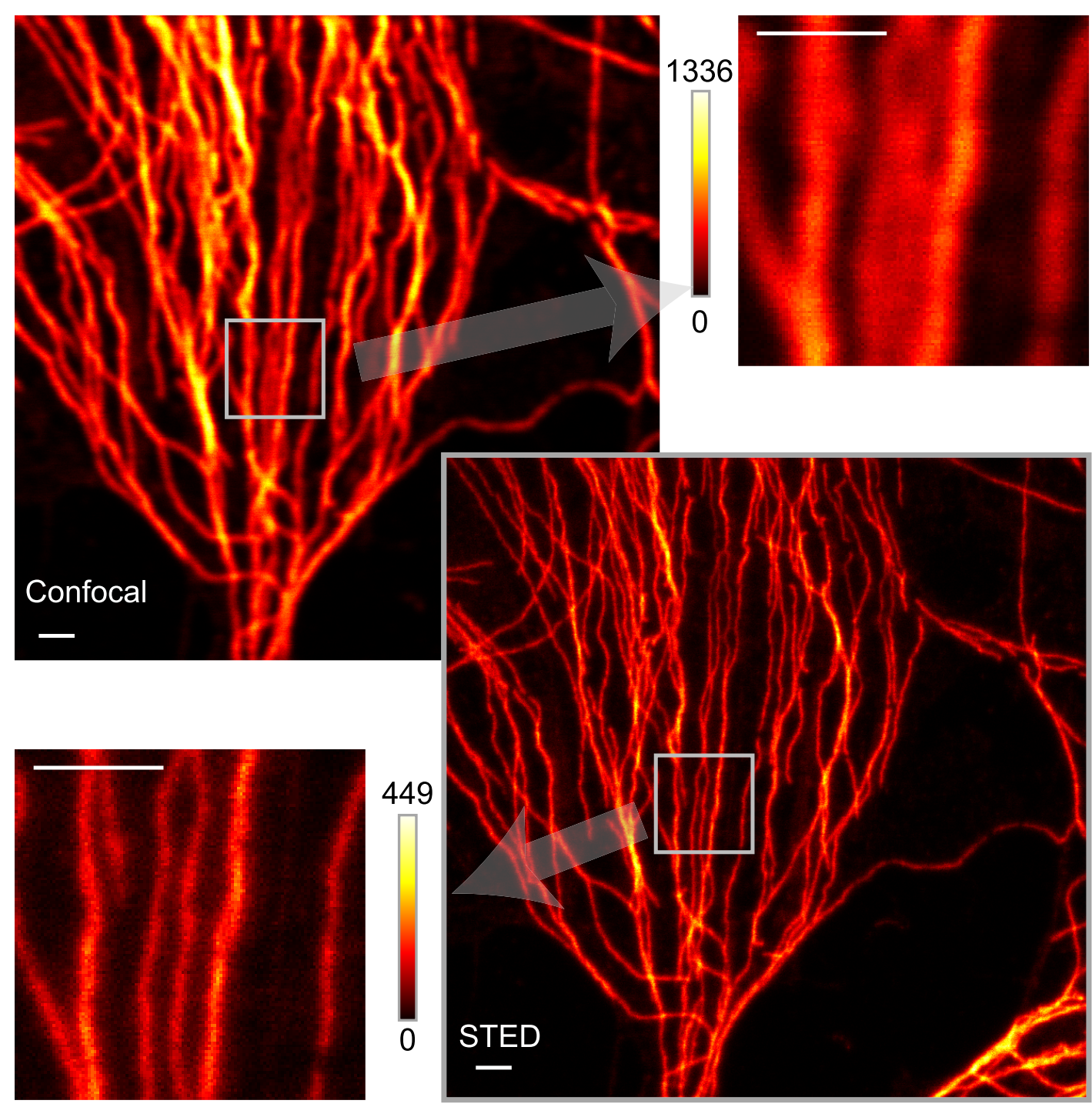

Figure A.2: Microtubuli immunolabeled with Atto 647N. Dwell time $500 \mu \mathrm{s}$, pixel size $22.5 \mathrm{~nm}$ x $22.5 \mathrm{~nm}$. Scale bars $1 \mu \mathrm{m}$

\section{Cell Culture and Immunocytochemistry}

Lamin: The SH-SY5Y neuroblastoma cell line was grown as described previously ([78]). Cells were seeded on standard glass coverslips to a confluency of about $80 \%$. For immunostaining of the nuclear lamina cells were fixed with $3,7 \%$ PFA for $15 \mathrm{~min}$ followed by a 5min-treatment with Triton X-100. Before the incubation with the primary antibody, the cells were blocked in 1\%BSA in PBS for 5min. Anti-lamin B1 rabbit IgG (Abcam, Cambridge, UK) was used as primary antibody, anti-rabbit conjugated Atto 647N IgG (Atto-Tec, Siegen, Germany) as secondary antibody respectively. Both antibodies were diluted in block- 

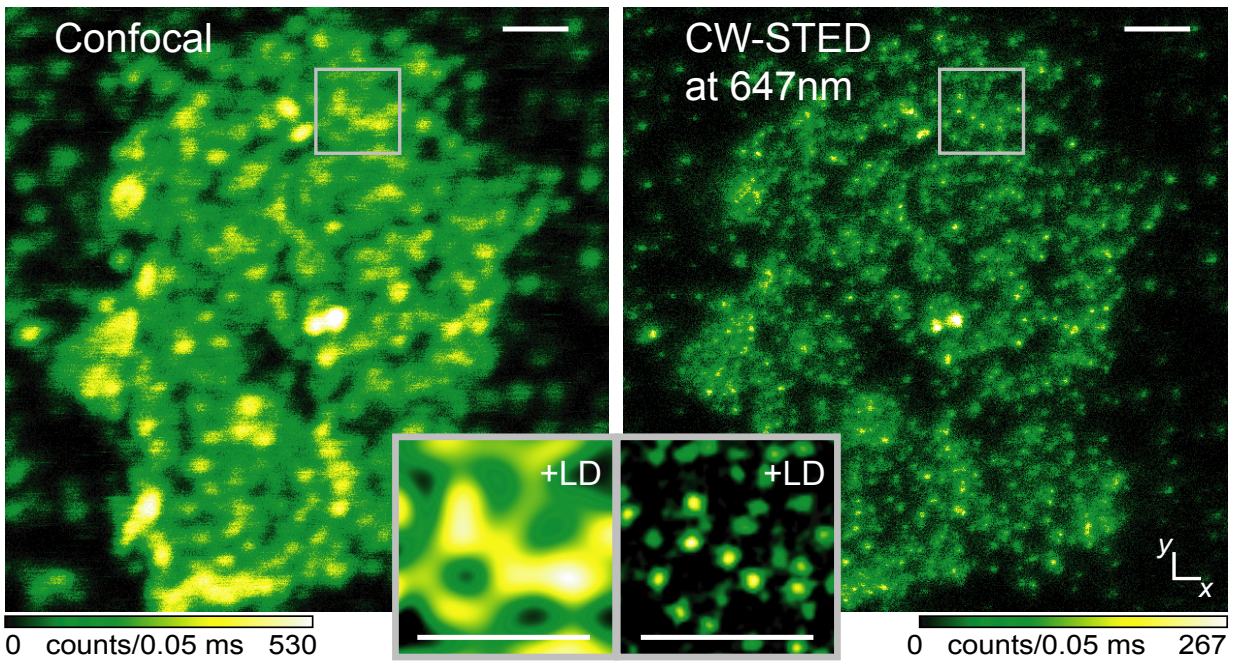

Figure A.3: CW STED microscopy of syntaxin clusters in cell membrane, immunolabeled with the dye Atto 565. $25 \mu \mathrm{W}$ excitation power (532 $\mathrm{nm}$ ) and $114 \mathrm{~mW}$ CW STED power $(647 \mathrm{~nm})$. Scale bars, $1 \mu \mathrm{m}$.

ing buffer. Cells were mounted in mowiol for the measurement shown in figure 4.6 and in water (with Triplet quencher) for the measurement shown in figure 3.10 .

Neurofilaments: The SH-SY5Y neuroblastoma cell line was grown as described 2. Cells were seeded on standard glass coverslips to a confluency of $\approx 80 \%$. All-trans-retinoic acid (RA) at $10 \mu M$ (EMD Biosciences, San Diego, CA) was added the day after plating. After 5 days in the presence of all-trans-retinoic acid [R2], cells were washed three times and incubated with $50 \mathrm{ng} / \mathrm{ml}$ human BDNF (Alomone Laboratories, Jerusalem, Israel) in serumfree medium. Medium containing BDNF was replaced every fifth day. After 2-4 weeks immunostaining of neurofilaments as a neuronal marker protein was performed with anti200kD neurofilament-heavy subunit mouse IgG (Abcam, Cambridge, U.K.) as the primary antibody and with Atto 647N (Atto-Tec, Siegen, Germany) conjugated sheep anti-mouse IgG as the secondary one. The cells were mounted in Mowiol. 


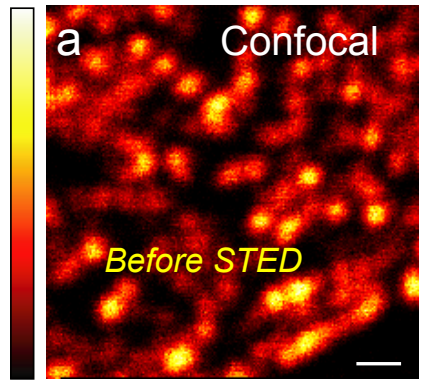

Total counts: 765,087

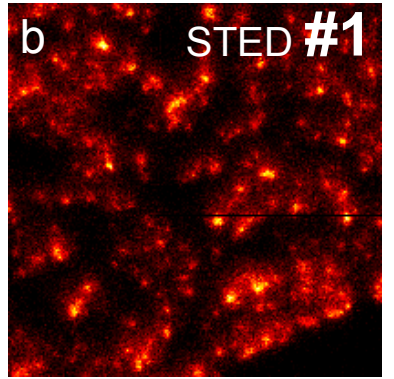

307,485

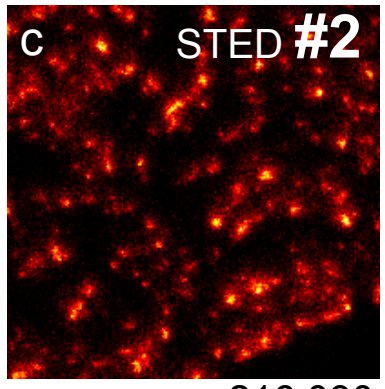

216,030

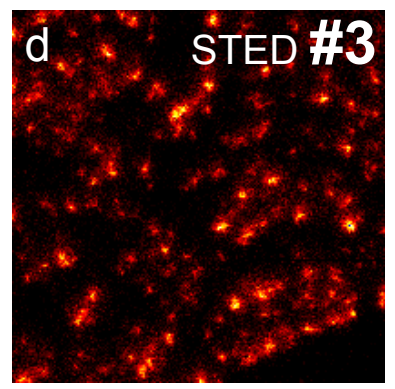

156,031

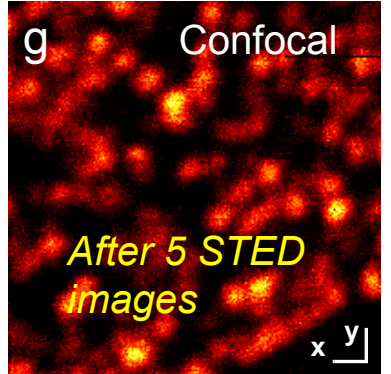

376,758

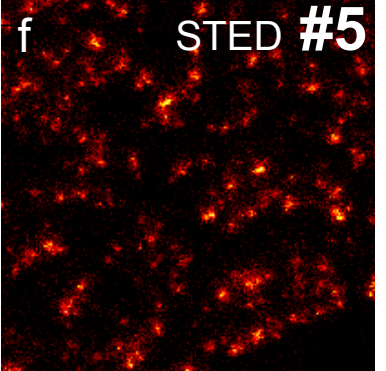

94,274

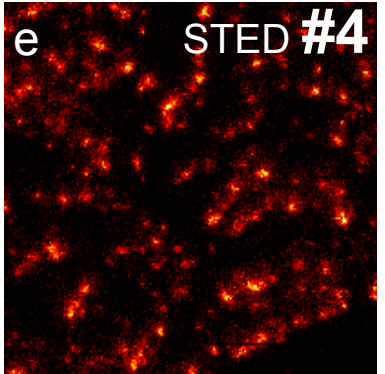

118,956

Figure A.4: Image sequence of syntaxin 1 on plasma membrane of neuroblastoma cells immunolabeled with Atto 565. The total counts are corrected for background noise. Pixel size: $20 \mathrm{~nm} \times 20 \mathrm{~nm}$. Pixel dwell time: $50 \mu \mathrm{s}$. Scale bar $500 \mathrm{~nm}$. 


\section{B List of Publications}

Parts of this thesis have been published as follows:

1. K. I. Willig*, B. Harke*, R. Medda, S. W. Hell. STED microscopy with continuous wave beams. Nature Methods 4 (11), 915 - 918, 2007.

*These authors contributed equally to this work

2. B. Harke*, C. Ullal*, J. Keller, S. W. Hell. Three-Dimensional Nanoscopy of Colloidal Crystals. Nano letters, DOI: 10.1021/n1073164n, 2008.

*These authors contributed equally to this work

3. B. Harke, J. Keller, C. K. Ullal, V. Westphal, A. Schönle, S. W. Hell. Resolution scaling in STED microscopy. Optics Express, Submitted 2008

4. J. J. Sieber, K. I. Willig, C. Kutzner, C. Gerding-Reimers, B. Harke, G. Donnert, B. Rammner, C. Eggeling, S. W. Hell, H. Grubmüller, T. Lang. Anatomy and dynamics of a supramolecular membrane protein cluster. Science 317, 1072-1076, 2007

5. A. Schönle, J. Keller, B. Harke, and S. Hell. Diffraction Unlimited Far-Field Fluorescence Microscopy. Handbook of Biological Nonlinear Optical Microscopy, M. Masters and P. So, Oxford University Press, in press 2008 



\section{Acknowledgment}

I would like to thank all the people who have contributed to the success of this thesis.

Prof. Dr. Stefan W. Hell not only proposed the fascinating project of 3D STED but also provided an outstanding scientific environment. His continuous input of new ideas has always encouraged me and contributed a lot to the success of this thesis.

Prof. Dr. Markus Münzenberg for his interest and being one of the referees of this thesis.

Dr. Volker Westphal for his assistance during my first years.

Dr. Jan Keller for a lot of help and assistance during several projects and for critical proofreading the manuscript.

Dr. Chaitanya K. Ullal for fancy colloidal crystals, nice discussions and proofreading the manuscript.

Dr Katrin I. Willig for the collaboration during the CW STED project, valuable discussions and proofreading the manuscript.

Rebecca Medda for offering brilliant biological samples.

Dr. Stefan Jakobs and Sylvia Löbermann for biological support.

Dr. Andreas Schönle for providing the lab software and valuable discussions.

Dr. Lars Kastrup, Dr. Scott Irvine and Brian Rankin for kindly proofreading the manuscript.

Hannes Bock, Christian Ringemann, Dr. Christian Eggeling, Arnold Giske, Jonas Fölling and Stefan Bretschneider for fruitful monday morning discussions especially about out-of-science topics.

Dr. Gael Moneron and Brian Rankin for important discussions about all kind of optics.

Jaydev Jethwa for always offering assistance and proofreading the manuscript.

Harald Meyer for technical support.

This work would not have been possible without a great mechanical and optical workshop.

Many thanks to my colleagues for having a nice time.

I would also like to thank Carola and my parents for supporting me during the last years. 


\section{Lebenslauf}

\section{Persönliche Daten}

Name, Vorname

Harke, Benjamin

Geburtsdatum

08.08.1980

Geburtsort

Celle

Familienstand

ledig

Nationalität

deutsch

\section{Schulausbildung}

$1986-1990$

1990 - 1992

1992 - 1999
Grundschule, Eschede

Orientierungsstufe Burgstraße, Celle

Gymnasium Ernestinum, Celle

\section{Studium}

$1999-2001$

$2001-2004$

seit 2004
Grundstudium der Physik, Universität Hannover

Haupstudium der Physik, Universität Hannover

Promotion am Max-Planck-Institut für Biophysikalische

Chemie, Abteilung Nanobiophotonik

Göttingen, 2008 Benjamin Harke 